\title{
Diversity and Sources of Multiple Disease Resistance in Hordeum spontaneum
}

T. G. Fetch, Jr., Cereal Research Centre, Agriculture and Agri-Food Canada, Winnipeg, Manitoba R3T 2M9, Canada; B. J. Steffenson, Department of Plant Pathology, University of Minnesota, St. Paul, MN 55108, USA; and E. Nevo, Institute of Evolution, University of Haifa, Mt. Carmel, Haifa 31905, Israel

\begin{abstract}
Fetch, T. G., Jr., Steffenson, B. J., and Nevo, E. 2003. Diversity and sources of multiple disease resistance in Hordeum spontaneum. Plant Dis. 87:1439-1448.

Hordeum spontaneum, the progenitor of cultivated barley, is known to be a rich source of disease resistance genes. The objective of this study was to assess the diversity of $H$. spontaneum accessions from Israel and Jordan for their reaction to six fungal pathogens of importance to cultivated barley in the United States and Canada. Overall, a high level of macro-scale (across collection sites) and micro-scale (within a collection site) diversity for disease reaction was found in the 116 accessions of $H$. spontaneum evaluated at the seedling stage. Additionally, genetic heterozygosity for resistance loci was common in $H$. spontaneum. The frequency of resistance in accessions from Jordan and Israel was high for Septoria speckled leaf blotch (77 and $98 \%$, respectively), leaf rust (70 and 90\%), net blotch (72 and 68\%), and powdery mildew (58 and 70\%); intermediate for spot blotch (53 and 46\%); and low for stem rust (2 and 26\%). The level of disease resistance in $H$. spontaneum was not strongly correlated with any of the weather variables (temperature, precipitation, and humidity) monitored near the collection sites. However, in general, resistance was more often found in germ plasm from mesic (e.g., Mediterranean coast) than in xeric (e.g., Negev Desert) areas. Two H. spontaneum accessions (Shechem 12-32 and Damon 11-11) were resistant to all six pathogens and may be useful parents in programs breeding barley for multiple disease resistance. The high level of diversity and heterozygosity for disease reaction found in this study indicates that $H$. spontaneum is an extraordinarily rich and largely untapped source of unique disease resistance alleles for cultivated barley improvement.
\end{abstract}

Additional keywords: Hordeum vulgare, wild barley

The Upper Midwest region of the United States and the central provinces of Canada comprise one of the largest barley (Hordeum vulgare L.) growing regions in North America. Diseases that commonly impact barley production in this region include stem rust (caused by Puccinia graminis Pers.:Pers. f. sp. tritici Eriks. \& E. Henn.), net blotch (caused by Pyrenophora teres Drechs f. teres Smedeg. [anamorph: Drechslera teres f. teres (Sacc.) Shoem.]), spot blotch (caused by Cochliobolus sativus (Ito \& Kurib.) Drechs. ex Dastur [anamorph: Bipolaris sorokiniana (Sacc.) Shoem.]), Septoria speckled leaf blotch (SSLB) (caused by Septoria passerinii Sacc.), and to a lesser degree leaf rust (caused by Puccinia hordei G. Otth) and powdery mildew (caused by Blumeria [=Erysiphe $]$ graminis (DC.) E.O. Speer f.

Corresponding author: T. G. Fetch, Jr.

E-mail: tfetch@agr.gc.ca

Published as paper number 1850 of the contribution series from the Cereal Research Centre of Agriculture and Agri-Food Canada.

Accepted for publication 14 July 2003.

Publication no. D-2003-0930-02R

(C) 2003 Department of Agriculture and Agri-

Food, Government of Canada sp. hordei Ém. Marchal) (39,41). The deployment of host resistance is often the preferred method of control for these diseases because it is an effective, economical, and environmentally sound strategy.

Although sources of resistance to these diseases have been identified in $H$. vulgare, they do not represent the greatest potential diversity available for breeding purposes. An alternative and rich source of genetic diversity for disease resistance is the wild barley species $H$. spontaneum (syn. $H$. vulgare L. subsp. spontaneum (C. Koch) Thell.). $H$. spontaneum is native to the Fertile Crescent $(32,50)$, a large region extending from Israel and western Jordan to southeastern Turkey and arcing down through eastern Iraq and western Iran (12). In this region, the host and its pathogens have co-evolved over thousands of years (3), resulting in a high degree of diversity for both resistance in the host and virulence in the pathogen. Indeed, previous investigators have identified numerous sources of resistance in $H$. spontaneum to powdery mildew $(11,15,31)$, leaf rust $(17,27)$, net blotch $(14,22,38)$, SSLB $(22,28,45)$, and leaf scald (1).

The center of origin for wild progenitor species is often the place where the greatest genetic diversity may be found. With respect to $H$. spontaneum, the region of the Fertile Crescent bounded by Israel and
Jordan may contain the greatest genetic diversity for the species $(4,32)$. Disease resistance is common in $H$. spontaneum; thus, it may be possible to identify individual accessions with resistance to all of the important diseases in the Upper Midwest region of the United States and central provinces of Canada. These accessions could then be crossed with advanced barley breeding lines to simultaneously transfer genes for multiple disease resistance and also increase allelic diversity. Thus, the objective of this study was to assess the diversity of $H$. spontaneum accessions from Israel (including Palestinian authority lands of the West Bank and the Gaza Strip) and Jordan for their reaction to six fungal pathogens and to identify sources of multiple disease resistance. A preliminary report of this research has been published (10).

\section{MATERIALS AND METHODS}

Plant materials. One hundred and sixteen $H$. spontaneum accessions from the Institute of Evolution, University of Haifa, Israel, were evaluated for resistance to six foliar pathogens in the greenhouse. Each accession was derived from a single plant and was arbitrarily selected from 57 collection sites in Israel and Jordan (Fig. 1). The accessions at each site were collected no closer than $1 \mathrm{~m}$ apart along a transect of 25 to $200 \mathrm{~m}$. Collection sites were ecogeographically diverse and varied greatly with respect to humidity (34 to $65 \%$ mean annual relative humidity $(\mathrm{RH})$ at 1400 hours), precipitation (71 to $1,600 \mathrm{~mm}$ annually), temperature ( 1 to $16^{\circ} \mathrm{C}$ and 20 to $33^{\circ} \mathrm{C}$ mean annual temperature for January and August, respectively), and elevation ( -350 to $1,530 \mathrm{~m}$ below/above sea level) (Tables 1 and 2). Standard susceptible and resistant checks and differential host lines (if available) also were included in the experiments to verify the infection type (IT)/infection response (IR) and virulence pattern, respectively, to each pathogen $(8,18,21,40,45,52)$.

Pathogen isolates. Information on the isolate designation, source, and pathotype (if known) of each fungal pathogen used is given in Table 3. All pathogen isolates were collected from infected barley plants in the field with the exception of the $B$. graminis f. sp. hordei isolate, which was taken from an infected plant in the greenhouse. Pathogens were stored as dried urediniospores at $-80^{\circ} \mathrm{C}$ (rusts) or as conidiospores using a silica gel method (9), with the exception of the B. graminis $\mathrm{f}$. $\mathrm{sp}$. 


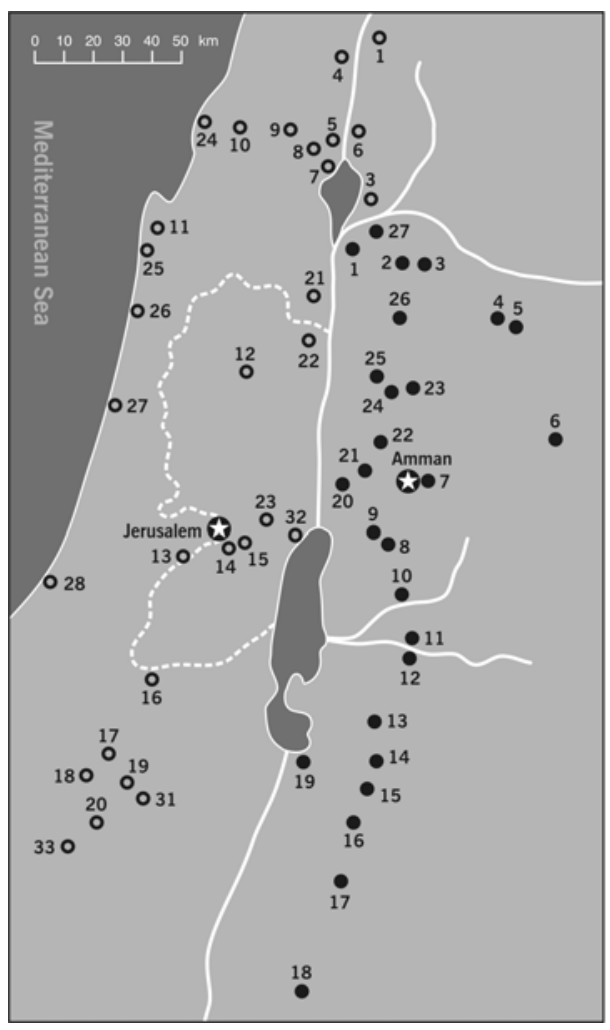

\begin{tabular}{|l|l|}
\hline O ISRAEL & - JORDAN \\
\hline 1 Mt. Hermon & 1 Shũna, N. \\
3 Afiq & 2 Irbid \\
4 Tel Hay & 3 Irbid, E. \\
5 Rosh Pinna & 4 Mafraq, W. \\
6 Gadot & 5 Mafraq \\
7 Tabigha & 6 Zarqa \\
8 Zefat & 7 Amman \\
9 Mt. Meron & 8 Madaba \\
10 Maalot & 9 Mt. Nebo \\
11 Damon & 10 Wadi Wala \\
12 Shechem & 11 Wadi Müjib \\
13 Bar Gliyyora & 12 Talal \\
14 Talpiyyot & 13 Karak \\
15 Eyzariya & 14 Wadi Hasa \\
16 Tel Shoket & 15 Laban \\
17 Bor Mashash & 16 Tafila \\
18 Revivum & 17 Danã \\
19 Yeroham & 18 Wadi Mũsã \\
20 Sede Boqer & 19 Safi \\
21 Bet Shean & 20 Shūna, S. \\
22 Mehola & 21 'Ira \\
23 Wadi Qilt & 22 Salt \\
24 Akhziv & 23 Jarash \\
25 Atilt & 24 Sakib \\
26 Caesarea & 25 Ajlun \\
27 Herziliyya & 26 Irbid, S. \\
28 Ashqelon & 27 El Hamma \\
31 Machtesh Gadol & \\
32 Ein Zukim & \\
33 Avedat & \\
\hline
\end{tabular}

Fig. 1. Geographic location of sampling sites of Hordeum spontaneum in Israel and Jordan. hordei isolate, which was from fresh plants. The pathogen isolates used represent common virulence spectra found in the Upper Midwest region of the United States. Two pathotypes of the stem rust pathogen were used in this study to more fully characterize the resistance spectra present in $H$. spontaneum. Pathotype QCCJ possesses virulence for the barley stem rust resistance gene Rpgl and avirulence for rpg4, while pathotype MCCF possesses avirulence for both Rpgl and rpg4 (18).

Inoculation protocols. For each disease evaluation, five seeds of each $H$. spontaneum accession were planted either in plastic pots ( $10 \mathrm{~cm}$ square) or in cones (3.8 $\mathrm{cm}$ diameter and $21 \mathrm{~cm}$ depth) filled with a peat moss:perlite (3:1 ratio) potting mixture. Fertilizer was applied at planting with water-soluble (15-0-15, N-P-K) and controlled release (14-14-14, N-P-K) formulations. Plants were grown in a greenhouse at $22 \pm 3^{\circ} \mathrm{C}$ with a 13 -h photoperiod provided by sunlight and metal halide bulbs (530 to $\left.710 \mu \mathrm{E} \cdot \mathrm{m}^{-2} \cdot \mathrm{s}^{-1}\right)$. For leaf and stem rust evaluations, 7-day-old seedlings (primary leaf fully expanded) were inoculated with heat-shocked urediniospores suspended in a lightweight mineral oil (4 $\mathrm{mg}$ of spores in $0.7 \mathrm{ml}$ of oil per 100 plants). Plants were then placed in mist (generated by ultrasonic humidifiers) chambers and incubated

Table 1. Ecogeographical data for collection sites of Hordeum spontaneum from Israel ${ }^{\mathrm{a}}$

\begin{tabular}{|c|c|c|c|c|c|c|c|c|}
\hline & \multirow[b]{2}{*}{ Location } & \multirow[b]{2}{*}{ Longitude } & \multirow[b]{2}{*}{ Latitude } & \multirow[b]{2}{*}{ Altitude $^{c}$} & \multicolumn{2}{|c|}{ Mean temperature $^{\text {b }}$} & \multirow{2}{*}{$\begin{array}{c}\text { Annual } \\
\text { rainfall }^{\mathrm{d}}\end{array}$} & \multirow{2}{*}{$\begin{array}{c}\text { Mean } \\
\text { humidity }\end{array}$} \\
\hline & & & & & Jan. & Aug. & & \\
\hline 1. & Mt. Hermon & 35.45 & 33.17 & 1,530 & 1 & 20 & 1,600 & 52 \\
\hline 3. & Afiq & 35.42 & 32.47 & 325 & 11 & 27 & 450 & 41 \\
\hline 4. & Tel Hay & 35.33 & 33.14 & 400 & 8 & 25 & 769 & 50 \\
\hline 5. & Rosh Pinna & 35.31 & 32.57 & 700 & 9 & 25 & 697 & 48 \\
\hline 6. & Gadot & 35.37 & 33.01 & 100 & 11 & 28 & 500 & 45 \\
\hline 7. & Tabigha & 35.32 & 32.52 & 0 & 15 & 32 & 436 & 45 \\
\hline 8. & Zefat & 35.30 & 32.56 & 800 & 8 & 24 & 718 & 48 \\
\hline 9. & Mt. Meron & 35.24 & 33.00 & 1,150 & 6 & 22 & 1,010 & 49 \\
\hline 10. & Maalot & 35.16 & 33.00 & 500 & 8 & 23 & 785 & 50 \\
\hline 11. & Damon & 35.00 & 32.44 & 425 & 11 & 24 & 686 & 59 \\
\hline 12. & Shechem & 35.14 & 32.14 & 400 & 9 & 24 & 618 & 46 \\
\hline 13. & Bar Giyyora & 35.05 & 31.43 & 760 & 10 & 26 & 537 & 49 \\
\hline 14. & Talpiyyot & 35.14 & 31.45 & 800 & 9 & 24 & 486 & 50 \\
\hline 15. & Eyzariya & 35.16 & 31.46 & 600 & 10 & 26 & 380 & 48 \\
\hline 16. & Tel Shoket & 34.55 & 31.19 & 375 & 11 & 26 & 280 & 45 \\
\hline 17. & Bor Mashash & 34.50 & 31.04 & 350 & 10 & 27 & 150 & 38 \\
\hline 18. & Revivum & 34.45 & 31.01 & 320 & 10 & 27 & 130 & 38 \\
\hline 19. & Yeroham & 34.54 & 30.59 & 490 & 10 & 26 & 130 & 35 \\
\hline 20. & Sede Boqer & 34.47 & 30.52 & 450 & 9 & 26 & 91 & 36 \\
\hline 21. & Bet Shean & 35.30 & 32.30 & -120 & 13 & 30 & 300 & 40 \\
\hline 22. & Mehola & 35.29 & 32.24 & -150 & 13 & 30 & 270 & 34 \\
\hline 23. & Wadi Qilt & 35.23 & 31.50 & 50 & 14 & 30 & 170 & 40 \\
\hline 24. & Akhziv & 35.06 & 33.03 & 10 & 12 & 26 & 620 & 60 \\
\hline 25. & Atlit & 34.57 & 32.42 & 50 & 13 & 26 & 500 & 65 \\
\hline 26. & Caesarea & 34.54 & 32.30 & 10 & 13 & 26 & 539 & 65 \\
\hline 27. & Herzliyya & 34.48 & 32.10 & 25 & 13 & 26 & 530 & 65 \\
\hline 28. & Ashqelon & 34.36 & 31.38 & 50 & 14 & 27 & 424 & 64 \\
\hline 31. & Machtesh Gadol & 34.58 & 30.58 & 600 & 9 & 26 & 170 & 35 \\
\hline 32. & Ein Zukim & 35.26 & 31.44 & -200 & 15 & 31 & 210 & 35 \\
\hline 33. & Avedat & 34.46 & 30.49 & 525 & 9 & 25 & 168 & 36 \\
\hline
\end{tabular}

${ }^{a}$ Meteorological data provided is from Nevo et al. 1984 (34).

${ }^{\mathrm{b}}$ Mean temperature is the average temperature $\left({ }^{\circ} \mathrm{C}\right)$ for January and August.

${ }^{c}$ Altitude is in meters above or below mean sea level.

${ }^{d}$ Annual rainfall is in $\mathrm{mm}$ of precipitation.

${ }^{\mathrm{e}}$ Mean humidity is the average relative humidity at $1400 \mathrm{~h}$ on an annual basis. 
at $20^{\circ} \mathrm{C}$ for $16 \mathrm{~h}$ in the dark at near $100 \%$ RH. After the mist period, leaf rust inoculated plants were allowed to slowly dry for at least $3 \mathrm{~h}$ before being placed in a growth chamber at 19 to $20^{\circ} \mathrm{C}$. Stem rust inoculated plants were exposed to light (120 to $160 \mu \mathrm{E} \cdot \mathrm{m}^{-2} \cdot \mathrm{s}^{-1}$ and a temperature ramp increasing from 20 to $24^{\circ} \mathrm{C}$ ) after the mist period and allowed to slowly dry for at least $3 \mathrm{~h}$ before being placed into one of two growth chamber environments (19 to $20^{\circ} \mathrm{C}$ or 27 to $28^{\circ} \mathrm{C}$ ). The two incubation environments were used because some stem rust resistance genes are known to be temperature sensitive (18). For net and spot blotch evaluations, inoculum was produced on agar plates seeded with silica gel crystals containing adsorbed conidia. Seedlings were inoculated ( 5 to $6 \times 10^{4}$ conidia per $\mathrm{ml}$ of $\mathrm{H}_{2} \mathrm{O}, 1.7 \times 10^{-1} \mathrm{ml}$ per plant) when the third leaf was fully expanded. Plants were placed in mist chambers overnight at $20^{\circ} \mathrm{C}$ and then incubated in a greenhouse at $22 \pm 3^{\circ} \mathrm{C}$. For powdery mildew evalua- tions, seedlings were inoculated at the primary leaf stage by shaking mildewinfected plants (previously infected with a single pustule isolate) over the $H$. spontaneum accessions and then incubating at ambient greenhouse temperature (23 \pm $2^{\circ} \mathrm{C}$ ) (41). For SSLB evaluations, accessions were inoculated $\left(5 \times 10^{5}\right.$ pycnidiospores per $\mathrm{ml}$ of $\mathrm{H}_{2} \mathrm{O}, 5.0 \times 10^{-1} \mathrm{ml}$ per plant) when the second leaf was fully expanded and then subjected to a 72-h mist period at $21^{\circ} \mathrm{C} /$ dark and $25^{\circ} \mathrm{C} /$ light (45). The first $40 \mathrm{~h}$ were in darkness, followed by a photoperiod of $5 \mathrm{~h}$ for the remainder of the mist period. Plants were then incubated in a greenhouse at $22 \pm 3^{\circ} \mathrm{C}$. All experiments were repeated once.

Disease assessment. Disease reactions were assessed after specific incubation periods using published assessment guides for each respective pathogen. Leaf and stem rust reactions were rated 10 to 13 days postinoculation using scales modified from Levine and Cherewick (24) and
Miller and Lambert (29), respectively. Powdery mildew was rated 9 to 10 days postinoculation using the 0 to 4 scale of Torp et al. (44). Net and spot blotch reactions were rated 10 to 12 days postinoculation using the 1 to 9 scales of Tekauz (43) and Fetch and Steffenson (9), respectively. SSLB was rated 17 to 18 days postinoculation using a 0 to 5 scale (45). For the rusts, powdery mildew, and SSLB, ITs/IRs of 0 or 1 were considered indicative of resistance, 2 of intermediate resistance, and 3 or higher of susceptibility. For net and spot blotch, IRs of 1 to 3 were considered indicative of resistance, 4 to 5 of intermediate resistance, and 6 to 9 of susceptibility.

Statistical analyses. Phenotypic diversity for disease reaction in $H$. spontaneum was estimated using the Shannon information index, where $h_{s \cdot j}=-\Sigma p_{i} \ln \left(p_{i}\right)$, with maximum $h_{s \cdot j}=1.099$ (16). For this analysis, the ITs/IRs for each disease were separated into the general reaction classes of resistant, intermediate, and susceptible as

Table 2. Ecogeographical data for collection sites of Hordeum spontaneum from Jordan ${ }^{\mathrm{a}}$

\begin{tabular}{|c|c|c|c|c|c|c|c|c|}
\hline & \multirow[b]{2}{*}{ Location } & \multirow[b]{2}{*}{ Longitude } & \multirow[b]{2}{*}{ Latitude } & \multirow[b]{2}{*}{ Altitude $^{c}$} & \multicolumn{2}{|c|}{ Mean temperature ${ }^{\mathrm{b}}$} & \multirow{2}{*}{$\begin{array}{l}\text { Annual } \\
\text { rainfall }^{\mathrm{d}}\end{array}$} & \multirow{2}{*}{$\begin{array}{c}\text { Mean } \\
\text { humidity }\end{array}$} \\
\hline & & & & & Jan. & Aug. & & \\
\hline 1. & Shūna, N. & 35.37 & 32.38 & -200 & 13 & 29 & 300 & 52 \\
\hline 2. & Irbid & 35.51 & 32.33 & 425 & 9 & 25 & 300 & 53 \\
\hline 3. & Irbid, E. & 35.59 & 32.32 & 340 & 9 & 25 & 250 & 53 \\
\hline 4. & Mafraq, W. & 36.09 & 32.24 & 625 & 8 & 24 & 100 & 56 \\
\hline 5. & Mafraq & 36.11 & 32.14 & 745 & 7 & 24 & 100 & 56 \\
\hline 6. & Zarqa & 36.22 & 32.03 & 511 & 9 & 26 & 142 & 52 \\
\hline 7. & Amman & 35.57 & 31.57 & 750 & 8 & 25 & 300 & 49 \\
\hline 8. & Madaba & 35.48 & 31.43 & 785 & 8 & 24 & 358 & 55 \\
\hline 9. & Mt. Nebo & 35.44 & 31.45 & 755 & 8 & 24 & 350 & 55 \\
\hline 10. & Wadi Wala & 35.47 & 31.33 & 450 & 10 & 26 & 200 & 51 \\
\hline 11. & Wadi Mūjib & 35.48 & 31.27 & 138 & 10 & 26 & 266 & 52 \\
\hline 12. & Talal & 35.49 & 31.26 & 145 & 10 & 24 & 145 & 60 \\
\hline 13. & Karak & 35.42 & 31.11 & 1,000 & 8 & 24 & 258 & 53 \\
\hline 14. & Wadi Hasa & 35.44 & 30.58 & 900 & 7 & 23 & 250 & 51 \\
\hline 15. & Laban & 35.58 & 30.55 & 825 & 8 & 25 & 250 & 51 \\
\hline 16. & Tafila & 35.36 & 30.50 & 1,000 & 7 & 23 & 250 & 49 \\
\hline 17. & Danā & 35.36 & 30.39 & 1,273 & 7 & 24 & 300 & 49 \\
\hline 18. & Wadi Mūsā & 35.29 & 30.19 & 1,100 & 4 & 20 & 229 & 54 \\
\hline 19. & Safi & 35.27 & 31.03 & -350 & 16 & 33 & 71 & 47 \\
\hline 20. & Shūna, S. & 35.38 & 31.54 & -230 & 15 & 32 & $<100$ & 49 \\
\hline 21. & 'Ira & 35.40 & 32.00 & 650 & 8 & 25 & 400 & 54 \\
\hline 22. & Salt & 35.43 & 32.02 & 850 & 6 & 22 & 500 & 57 \\
\hline 23. & Jarash & 35.54 & 32.16 & 585 & 6 & 22 & 500 & 60 \\
\hline 24. & Sakib & 35.47 & 32.16 & 1,000 & 6 & 23 & 500 & 58 \\
\hline 25. & Ajlun & 35.45 & 32.20 & 1,150 & 6 & 23 & 600 & 61 \\
\hline 26. & Irbid, S. & 35.51 & 32.27 & 785 & 9 & 25 & 400 & 58 \\
\hline 27. & El Hamma & 35.41 & 32.42 & -75 & 13 & 30 & 377 & 60 \\
\hline
\end{tabular}

${ }^{a}$ Meteorological data provided courtesy of Faisal Nimer.

${ }^{\mathrm{b}}$ Mean temperature is the average temperature $\left({ }^{\circ} \mathrm{C}\right)$ for January and August.

${ }^{c}$ Altitude is in meters above or below mean sea level.

${ }^{\mathrm{d}}$ Annual rainfall is in $\mathrm{mm}$ of precipitation.

${ }^{\mathrm{e}}$ Mean humidity is the average relative humidity at $1400 \mathrm{~h}$ on an annual basis.

Table 3. Isolate designation, source, and pathotype of fungal pathogens used to evaluate resistance in Hordeum spontaneum

\begin{tabular}{|c|c|c|c|c|}
\hline Pathogen & Isolate & Source & Pathotype & Citation for pathotype name \\
\hline Puccinia graminis f. sp. tritici & A-1 & J. Miller & MCCF & Roelfs and Martens, 1988 (37) \\
\hline Puccinia graminis f. sp. tritici & QCC-2 & J. Miller & QCCJ & Roelfs and Martens, 1988 (37) \\
\hline Pyrenophora teres f. teres & ND89-19 & B. Steffenson & $1-2-6-7-10-13-16-18-25$ & Steffenson and Webster, 1992 (42); Wu et al., 2003 (52) \\
\hline Cochliobolus sativus & ND85F & B. Steffenson & 1 & Valjavec and Steffenson, 1997 (47) \\
\hline Septoria passerinii & ND97-15 & B. Steffenson & Not established & Not established \\
\hline Blumeria graminis f. sp. hordei & ND99-1 & B. Steffenson & 2.0.0.0.5.5.0.6 & Limpert and Muller, 1994 (25) \\
\hline Puccinia hordei & ND8702 & Y. Jin & MCJ & Long et al., 1995 (26) \\
\hline
\end{tabular}


previously described. Shannon's information index $\left(h_{s . j}\right)$ was determined based on the frequency $\left(p_{i}\right)$ of each class. Chi-square values were calculated using contingency tables $(2 \times 3)$ to estimate the homogeneity between populations derived from Israel and Jordan for each disease. Pearson correlation coefficients (PROC CORR, SAS version 8 , SAS Institute, Cary, NC) were used to assess the relationship between the average infection response (IR/IT) of each accession and the environmental parameters (i.e., those most likely to affect disease pressure) at each $H$. spontaneum collection site. For net and spot blotch, the actual IR was used for correlation, while for the other diseases the IR/IT was adjusted from a 0 to 4 scale to a 1 to 5 scale (by adding 1) for statistical purposes.

\section{RESULTS}

The reactions of control and differential lines were consistent with previously published results $(8,18,21,40,45,52)$. Accessions of $H$. spontaneum exhibited similar, but not identical ITs/IRs in the two experiments. The most common ITs/IRs observed on $H$. spontaneum across the two experiments are presented in Tables 4 and 5.

The highest frequency of disease resistance found in $H$. spontaneum was to $S$. passerinii. Over $98 \%$ of accessions from Israel (Table 4; all but Eyzariya 15-29) and $77 \%$ of accessions from Jordan (Table 5) exhibited IRs of 0 to 2 to $S$. passerinii. Most accessions (83\% from Israel and 57\% from Jordan) exhibited an IR of 0, indicating a very high level of resistance. Resistant and susceptible accessions were found within individual collection sites in both Israel (Eyzariya) and Jordan (N. Shūna, Wadi Mūjib, Talal, Karak, Laban, Tafila, S. Shūna, and Jarash). This indicates that micro-scale diversity exists for $S$. passerinii reaction at some locations.

Accessions of $H$. spontaneum with resistance (ITs of 0 to 2 ) to powdery mildew and leaf rust also were very common in both Israel (Table 4; 70 and 90\%, respectively) and Jordan (Table 5; 58 and 70\%, respectively). Highly resistant (IR of 0 or IT of 0;) accessions comprised 41 and $44 \%$ of the total from Israel and 36 and $13 \%$ of the total from Jordan for the two respective diseases. Resistant and susceptible accessions were found at a number of collection sites in both Israel (Gadot, Shechem, Bar Giyyora, Eyzariya, Tel Shoket, Bor Mashash, Yeroham, Wadi Qilt, Ein-Zukim, and Avedat) and Jordan (Irbid, East Irbid, West Mafraq, Amman, Madaba, Mt. Nebo, Wadi Mūjib, Talal, Laban, Tafila, Wadi Mūsa, Safi, South Shūna, Salt, Sakib, and S. Irbid), indicating micro-scale diversity for reaction to the two diseases. Plants resistant and susceptible to powdery mildew were found within several accessions from Israel (Gadot 6-35, Eyzariya 15-29, and Ein-zukim 32-05). The same was true for leaf rust with accessions Yeroham 1932, Sede Boqer 20-22, and Machtesh 3113. This result suggests heterozygosity for powdery mildew and leaf rust resistance loci in these single plant-derived accessions.

Resistance to net blotch also was frequent in $H$. spontaneum, as about $70 \%$ of accessions from both Israel (Table 4) and Jordan (Table 5) exhibited IRs of 1 to 5. Approximately one-third of the accessions from both countries were highly resistant (IRs of 1 to 2). Resistant and susceptible accessions were found at 11 of the $30 \mathrm{col}-$ lection sites in Israel and 15 of the 27 collection sites in Jordan. This indicates that micro-scale diversity for net blotch reaction exists across a number of locations in both countries, especially Jordan. Resistant and susceptible plants were detected within three accessions (Laban 15-09, Danā 1748, and Ajlun 25-35) from Jordan, suggesting heterozygosity at net blotch resistance loci.

Resistance to spot blotch was less common in $H$. spontaneum, as only 46 and $53 \%$ of accessions from Israel (Table 4) and Jordan (Table 5), respectively, exhibited IRs of 1 to 5. Only six highly resistant (IRs of 1 to 2) accessions were found from the two countries. Resistant and susceptible accessions were found at 14 of the 30 collection sites in Israel and 10 of the 27 collection sites in Jordan, again indicating micro-scale diversity for spot blotch reaction across a number of locations. Resistant and susceptible plants also were detected within two accessions (Tabigha 7-12 and Eyzariya 15-29) from Israel, suggesting heterozygosity at spot blotch resistance loci.

The lowest frequency of disease resistance in $H$. spontaneum was to stem rust. Only 26 and $11 \%$ of the accessions from Israel exhibited ITs of 0 ; to $2^{+}$to pathotypes MCCF and QCCJ, respectively, at the low incubation temperature (Table 4). The resistance in most of these accessions was temperature sensitive, as only 4 of 16 and 1 of 7 accessions remained resistant to pathotypes MCCF and QCCJ, respectively, at the high incubation temperature. Shechem 12-37 was the only highly resistant (IT $=0$;) accession identified (pathotype MCCF at low temperature). Accessions Mt. Hermon 1-46, Gadot 6-35, Zefat 8-05, Damon 11-11, Shechem 12-32, Tel Shoket 16-46, and Revivum 18-04 exhibited some resistance to both pathotypes at the low incubation temperature. Resistance to stem rust was rare in $H$. spontaneum accessions from Jordan, as only three accessions (Zarqa 6-21, Madaba 8-32, Wadi Wala 1005) exhibited ITs of $2^{-}$to $2^{+}$to pathotype MCCF or QCCJ under either of the two incubation environments (Table 5). Zarqa 6-21 was the only accession that was resistant to both pathotypes (low incubation temperature only). Resistant and susceptible accessions to at least one of the patho- types were found at 12 of the 30 collection sites in Israel and three of the 27 collection sites in Jordan. Resistant and susceptible plants also were detected within five accessions (Rosh Pinna 5-23, Shechem 1237, Tel Shoket 16-46, Revivum 18-04, and Yeroham 19-32) from Israel to at least one of the two pathotypes, suggesting heterozygosity at stem rust resistance loci.

A high level of diversity $\left(h_{s . j}=0.993\right.$ to 1.025) for reaction to powdery mildew, leaf rust, net blotch, and spot blotch was found in the combined analysis for accessions from both Israel and Jordan (Table 6). The lowest level of diversity found was for reaction to SSLB and stem rust (pathotype $\mathrm{MCCF})\left(h_{s . j}=0.615\right.$ and 0.519 , respectively). Chi-square analysis using $2 \times$ 3 contingency tables revealed that the frequency of general disease reactions was similar between accessions from Israel and Jordan for powdery mildew $\left(\chi^{2}=1.64, P=\right.$ $0.45, \mathrm{NS})$, net blotch $\left(\chi^{2}=0.84, P>0.95\right.$, NS), and spot blotch $\left(\chi^{2}=1.67, P=0.45\right.$, NS). However, significant differences in class frequencies were found between accessions from the two countries for SSLB $\left(\chi^{2}=13.04, P<0.005\right)$ and leaf rust $\left(\chi^{2}=\right.$ 9.87, $P<0.01)$. These differences were attributed to a lower than expected frequency of susceptible responses for accessions from Israel and a higher than expected frequency of susceptible responses for accessions from Jordan for both SSLB and leaf rust. Since there were no values in the resistant class for stem rust reaction among the accessions from Jordan, a $2 \times 2$ contingency table was used. Significant differences in class frequencies were detected between accessions from Israel and Jordan for stem rust (MCCF, low temperature regime) $\left(\chi^{2}=5.61, P<0.025\right)$. These differences were mainly attributed to a higher than expected frequency of intermediate responses for accessions from Israel and a lower than expected frequency of intermediate responses for accessions from Jordan.

The relationship between environmental parameters near the collection sites and the general disease response of $H$. spontaneum accessions is presented in Table 7. In general, no consistent and statistically significant relationships were detected between the environmental parameters of altitude, temperature, rainfall, or humidity and the disease response of $H$. spontaneum. A low but significant correlation was found between the level of resistance to Puccinia hordei and the amount of rainfall and level of humidity in both Israel and Jordan. In Israel, resistance to B. graminis f. sp. hor$d e i$ was associated with high humidity and cooler daytime temperatures in August.

\section{DISCUSSION}

Overall, a high level of macro-scale diversity (i.e., across collection sites) for disease reaction was found in the 116 accessions of $H$. spontaneum evaluated. This 
Table 4. Disease reactions of Hordeum spontaneum accessions from Israel to Septoria passerinii, Blumeria graminis f. sp. hordei, Puccinia hordei, Pyrenophora teres f. teres, Cochliobolus sativus, and Puccinia graminis f. sp. tritici

\begin{tabular}{|c|c|c|c|c|c|c|c|c|c|c|c|c|c|c|c|c|c|c|}
\hline \multirow[b]{3}{*}{ Line } & \multirow{2}{*}{\multicolumn{2}{|c|}{$\begin{array}{c}\text { Septoria } \\
\text { passerinii }^{\mathrm{a}}\end{array}$}} & \multirow{2}{*}{\multicolumn{2}{|c|}{$\begin{array}{l}\text { B. g. f. sp. } \\
\text { hordei }^{\text {b }}\end{array}$}} & \multirow{2}{*}{\multicolumn{2}{|c|}{$\begin{array}{c}\text { Puccinia } \\
\text { hordei }^{\mathrm{c}}\end{array}$}} & \multirow{2}{*}{\multicolumn{2}{|c|}{$\begin{array}{l}\text { Pyrenophora } \\
\text { teres f. teres }\end{array}$}} & & & & & cinic & ramin & $s$ f. sp. & tritic & & \\
\hline & & & & & & & & & & $u s^{\mathrm{d}}$ & MCC & $10^{c}$ & MC & hic & QCC & $l^{c}$ & QCC & $\mathbf{J} \mathbf{h i}^{\mathbf{c}}$ \\
\hline & IR & IPe & IR & IP & IT & IP & IR & IP & IR & IP & IT & IP & IT & IP & IT & $\mathbf{I P}$ & IT & IP \\
\hline Mt. Hermon 1-13 & 0 & $\mathrm{R}$ & 2 & I & $1 n$ & $\mathrm{R}$ & 3 & $\mathrm{R}$ & 6 & $\mathrm{~S}$ & $3^{-}$ & $\mathrm{S}$ & $3^{-}$ & $\mathrm{S}$ & 3 & $\mathrm{~S}$ & $3^{+}$ & $\mathrm{S}$ \\
\hline Mt. Hermon 1-46 & 1 & $\mathrm{R}$ & 0 & $\mathrm{R}$ & $1 \mathrm{~N}$ & $\mathrm{R}$ & 8 & $\mathrm{~S}$ & 7 & $\mathrm{~S}$ & $1^{+}$ & $\mathrm{R}$ & $3^{-}$ & $\mathrm{S}$ & $2^{-}$ & I & 3 & $\mathrm{~S}$ \\
\hline Afiq 3-11 & 0 & $\mathrm{R}$ & 3 & $\mathrm{~S}$ & $0 ; \mathrm{n}$ & $\mathrm{R}$ & 8 & $\mathrm{~S}$ & 5 & I & 3 & $\mathrm{~S}$ & 3 & $\mathrm{~S}$ & 3 & $\mathrm{~S}$ & 3 & $\mathrm{~S}$ \\
\hline Afiq 3-23 & 0 & $\mathrm{R}$ & 3 & $\mathrm{~S}$ & $0 ; n$ & $\mathrm{R}$ & 8 & $\mathrm{~S}$ & 6 & $\mathrm{~S}$ & 3 & $\mathrm{~S}$ & 3 & $\mathrm{~S}$ & 3 & $\mathrm{~S}$ & $3^{-}$ & $\mathrm{S}$ \\
\hline Tel Hay 4-13 & 0 & $\mathrm{R}$ & 0 & $\mathrm{R}$ & $2 \mathrm{cn}$ & $\mathrm{I}$ & 6 & $\mathrm{~S}$ & 5 & $\mathrm{I}$ & $3 c$ & $\mathrm{~S}$ & 3 & $\mathrm{~S}$ & 3 & $\mathrm{~S}$ & $3 \mathrm{C}$ & $\mathrm{S}$ \\
\hline Tel Hay 4-19 & 1 & $\mathrm{R}$ & 1 & $\mathrm{R}$ & $2^{+} \mathrm{c}$ & I & 4 & $\mathrm{I}$ & 6 & $\mathrm{~S}$ & 3 & $\mathrm{~S}$ & 3 & $\mathrm{~S}$ & 3 & $\mathrm{~S}$ & $3 \mathrm{C}$ & $\mathrm{S}$ \\
\hline Tel Hay 4-43 & 0 & $\mathrm{R}$ & 0 & $\mathrm{R}$ & $1 \mathrm{cn}$ & $\mathrm{R}$ & 3 & $\mathrm{R}$ & 4 & $\mathrm{I}$ & $3 c$ & $\mathrm{~S}$ & 3 & $\mathrm{~S}$ & 3 & $\mathrm{~S}$ & $3 \mathrm{C}$ & $\mathrm{S}$ \\
\hline Rosh Pinna 5-23 & 1 & $\mathrm{R}$ & 0 & $\mathrm{R}$ & $0 ; \mathrm{c}$ & $\mathrm{R}$ & 8 & $\mathrm{~S}$ & 5 & $\mathrm{I}$ & $3^{-} \mathrm{c}$ & $\mathrm{S}$ & $3^{+}$ & $\mathrm{S}$ & $3 c$ & $\mathrm{~S}$ & $3^{+} / 2^{+}$ & $\mathrm{S} / \mathrm{I}$ \\
\hline Rosh Pinna 5-58 & 0 & $\mathrm{R}$ & 0 & $\mathrm{R}$ & $0 ; \mathrm{cn}$ & $\mathrm{R}$ & 8 & $\mathrm{~S}$ & 6 & $\mathrm{~S}$ & $3^{-} \mathrm{c}$ & $\mathrm{S}$ & $3^{+}$ & $\mathrm{S}$ & 3 & $\mathrm{~S}$ & 3 & $\mathrm{~S}$ \\
\hline Gadot 6-35 & 0 & $\mathrm{R}$ & $2 / 3$ & $\mathrm{I} / \mathrm{S}$ & $2 \mathrm{cn}$ & I & 7 & $\mathrm{~S}$ & 2 & $\mathrm{R}$ & $1^{+}$ & $\mathrm{R}$ & $2^{+}$ & I & $1^{+}$ & $\mathrm{R}$ & $3^{-}$ & $\mathrm{S}$ \\
\hline Gadot 6-45 & 0 & $\mathrm{R}$ & 4 & $\mathrm{~S}$ & $0 ; \mathrm{c}$ & $\mathrm{R}$ & 3 & $\mathrm{R}$ & 7 & $\mathrm{~S}$ & $3^{-}$ & $\mathrm{S}$ & 3 & $\mathrm{~S}$ & 3 & $\mathrm{~S}$ & $3^{-}$ & $\mathrm{S}$ \\
\hline Tabigha 7-06 & 0 & $\mathrm{R}$ & 2 & I & $2 \mathrm{C}$ & I & 3 & $\mathrm{R}$ & 5 & I & $3^{-}$ & $\mathrm{S}$ & $3^{+}$ & $\mathrm{S}$ & 3 & $\mathrm{~S}$ & 3 & $\mathrm{~S}$ \\
\hline Tabigha 7-12 & 0 & $\mathrm{R}$ & 2 & $\mathrm{I}$ & $2^{-} \mathrm{c}$ & $\mathrm{I}$ & 7 & $\mathrm{~S}$ & $3 / 6$ & $\mathrm{R} / \mathrm{S}$ & $3^{-} \mathrm{c}$ & $\mathrm{S}$ & 3 & $\mathrm{~S}$ & 3 & $\mathrm{~S}$ & 3 & $\mathrm{~S}$ \\
\hline Zefat 8-05 & 0 & $\mathrm{R}$ & 0 & $\mathrm{R}$ & $0 ; \mathrm{N}$ & $\mathrm{R}$ & 6 & $\mathrm{~S}$ & 6 & $\mathrm{~S}$ & $2^{-}$ & I & 3 & $\mathrm{~S}$ & $2^{+}$ & I & $3^{-}$ & $\mathrm{S}$ \\
\hline Zefat 8-11 & 0 & $\mathrm{R}$ & 0 & $\mathrm{R}$ & ln & $\mathrm{R}$ & 1 & $\mathrm{R}$ & 7 & $\mathrm{~S}$ & 3 & $\mathrm{~S}$ & $3^{-}$ & $\mathrm{S}$ & 3 & $\mathrm{~S}$ & 3 & $\mathrm{~S}$ \\
\hline Zefat 8-58 & 2 & $\mathrm{I}$ & 2 & $\mathrm{I}$ & $1 \mathrm{~N}$ & $\mathrm{R}$ & 2 & $\mathrm{R}$ & 4 & I & $3^{-} \mathrm{c}$ & $\mathrm{S}$ & $3^{-}$ & $\mathrm{S}$ & $3^{-} \mathrm{c}$ & $\mathrm{S}$ & $3^{-}$ & $\mathrm{S}$ \\
\hline Mt. Meron 9-09 & 0 & $\mathrm{R}$ & 0 & $\mathrm{R}$ & $2 \mathrm{c}$ & $\mathrm{I}$ & 3 & $\mathrm{R}$ & 6 & $\mathrm{~S}$ & 3 & $\mathrm{~S}$ & $3^{-}$ & $\mathrm{S}$ & $3^{-}$ & $\mathrm{S}$ & 3 & $\mathrm{~S}$ \\
\hline Mt. Meron 9-53 & 0 & $\mathrm{R}$ & 0 & $\mathrm{R}$ & $2 \mathrm{c}$ & I & 3 & $\mathrm{R}$ & 6 & $\mathrm{~S}$ & $2^{+}$ & $\mathrm{I}$ & $3^{-}$ & $\mathrm{S}$ & $3^{-}$ & $\mathrm{S}$ & 3 & $\mathrm{~S}$ \\
\hline Maalot 10-24 & 0 & $\mathrm{R}$ & 3 & $\mathrm{~S}$ & $0 ; n$ & $\mathrm{R}$ & 7 & $\mathrm{~S}$ & 6 & $\mathrm{~S}$ & $3^{-}$ & $\mathrm{S}$ & 3 & $\mathrm{~S}$ & $3^{-}$ & $\mathrm{S}$ & 3 & $\mathrm{~S}$ \\
\hline Maalot $10-36$ & 0 & $\mathrm{R}$ & 3 & $\mathrm{~S}$ & $0 ; \mathrm{n}$ & $\mathrm{R}$ & 8 & $\mathrm{~S}$ & 6 & $\mathrm{~S}$ & $3^{-}$ & $\mathrm{S}$ & 3 & $\mathrm{~S}$ & $3^{-}$ & $\mathrm{S}$ & 3 & $\mathrm{~S}$ \\
\hline Damon 11-02 & 0 & $\mathrm{R}$ & 0 & $\mathrm{R}$ & $0 ; \mathrm{c}$ & $\mathrm{R}$ & 2 & $\mathrm{R}$ & 5 & $\mathrm{I}$ & $1^{+}$ & $\mathrm{R}$ & $3^{-}$ & $\mathrm{S}$ & $3^{-}$ & $\mathrm{S}$ & 3 & $\mathrm{~S}$ \\
\hline Damon 11-11 & 0 & $\mathrm{R}$ & 0 & $\mathrm{R}$ & $0 ; \mathrm{cn}$ & $\mathrm{R}$ & 4 & $\mathrm{I}$ & 5 & $\mathrm{I}$ & 1 & $\mathrm{R}$ & $2^{-}$ & $\mathrm{I}$ & $2^{+}$ & $\mathrm{I}$ & $3^{-}$ & $\mathrm{S}$ \\
\hline Shechem 12-32 & 0 & $\mathrm{R}$ & 2 & $\mathrm{I}$ & $1^{+} \mathrm{cn}$ & $\mathrm{R}$ & 1 & $\mathrm{R}$ & 3 & $\mathrm{R}$ & $1^{+}$ & $\mathrm{R}$ & $3^{-}$ & $\mathrm{S}$ & $1^{+}$ & $\mathrm{R}$ & 3 & $\mathrm{~S}$ \\
\hline Shechem 12-37 & 0 & $\mathrm{R}$ & 3 & $\mathrm{~S}$ & $0 ; \mathrm{Nc}$ & $\mathrm{R}$ & 1 & $\mathrm{R}$ & 5 & $\mathrm{I}$ & $0 ; / 3^{-}$ & $\mathrm{R} / \mathrm{S}$ & $1^{+}$ & $\mathrm{R}$ & 3 & $\mathrm{~S}$ & $3^{-}$ & $\mathrm{S}$ \\
\hline Bar Giyyora 13-29 & 0 & $\mathrm{R}$ & 3 & $\mathrm{~S}$ & $0 ; \mathrm{N}$ & $\mathrm{R}$ & 2 & $\mathrm{R}$ & 5 & $\mathrm{I}$ & $3^{-}$ & $\mathrm{S}$ & 3 & $\mathrm{~S}$ & 3 & $\mathrm{~S}$ & 3 & $\mathrm{~S}$ \\
\hline Bar Giyyora 13-56 & 0 & $\mathrm{R}$ & 2 & $\mathrm{I}$ & $0 ; \mathrm{nc}$ & $\mathrm{R}$ & 4 & $\mathrm{I}$ & 6 & $\mathrm{~S}$ & $3^{-}$ & $\mathrm{S}$ & 3 & $\mathrm{~S}$ & $3^{-}$ & $\mathrm{S}$ & $3^{-}$ & $\mathrm{S}$ \\
\hline Talpiyyot $14-13$ & 0 & $\mathrm{R}$ & 1 & $\mathrm{R}$ & $0 ; \mathrm{N}$ & $\mathrm{R}$ & 6 & $\mathrm{~S}$ & 5 & $\mathrm{I}$ & 3 & $\mathrm{~S}$ & 3 & $\mathrm{~S}$ & $3^{+}$ & $\mathrm{S}$ & 3 & $\mathrm{~S}$ \\
\hline Talpiyyot $14-20$ & 0 & $\mathrm{R}$ & $0 / 2$ & $\mathrm{R} / \mathrm{I}$ & $0 ; \mathrm{N}$ & $\mathrm{R}$ & 5 & $\mathrm{I}$ & 5 & I & 3 & $\mathrm{~S}$ & 3 & $\mathrm{~S}$ & 3 & $\mathrm{~S}$ & 3 & $\mathrm{~S}$ \\
\hline Eyzariya $15-29$ & 5 & $\mathrm{~S}$ & $0 / 3$ & $\mathrm{R} / \mathrm{S}$ & $2 c$ & $\mathrm{I}$ & 5 & $\mathrm{I}$ & $3 / 6$ & $\mathrm{R} / \mathrm{S}$ & 3 & $\mathrm{~S}$ & 3 & $\mathrm{~S}$ & 3 & $\mathrm{~S}$ & 3 & $\mathrm{~S}$ \\
\hline Eyzariya $15-46$ & 0 & $\mathrm{R}$ & 1 & $\mathrm{R}$ & $3^{-} \mathrm{c}$ & $\mathrm{S}$ & 7 & $\mathrm{~S}$ & 4 & I & $3^{-}$ & $\mathrm{S}$ & $3^{+}$ & $\mathrm{S}$ & $3^{-}$ & $\mathrm{S}$ & 3 & $\mathrm{~S}$ \\
\hline Tel Shoket 16-05 & 0 & $\mathrm{R}$ & 2 & I & $1^{+} \mathrm{nc}$ & $\mathrm{R}$ & 2 & $\mathrm{R}$ & 6 & $\mathrm{~S}$ & $3^{-}$ & $\mathrm{S}$ & $3^{-}$ & $\mathrm{S}$ & $3^{-}$ & $\mathrm{S}$ & $3^{+}$ & $\mathrm{S}$ \\
\hline Tel Shoket 16-46 & 0 & $\mathrm{R}$ & 3 & $\mathrm{~S}$ & $0 ; \mathrm{n}$ & $\mathrm{R}$ & 2 & $\mathrm{R}$ & 7 & $\mathrm{~S}$ & $1^{+}$ & $\mathrm{R}$ & 3 & $\mathrm{~S}$ & $2^{+} / 3$ & $\mathrm{I} / \mathrm{S}$ & 3 & $\mathrm{~S}$ \\
\hline Bor Mashash 17-05 & 1 & $\mathrm{R}$ & 3 & $\mathrm{~S}$ & $2 \mathrm{c}$ & I & 2 & $\mathrm{R}$ & 5 & I & 3 & $\mathrm{~S}$ & 3 & $\mathrm{~S}$ & 3 & $\mathrm{~S}$ & 3 & $\mathrm{~S}$ \\
\hline Bor Mashash 17-11 & 0 & $\mathrm{R}$ & 3 & $\mathrm{~S}$ & $3^{-} \mathrm{C}$ & $\mathrm{S}$ & 3 & $\mathrm{R}$ & 6 & $\mathrm{~S}$ & 3 & $\mathrm{~S}$ & 3 & $\mathrm{~S}$ & 3 & $\mathrm{~S}$ & 3 & $\mathrm{~S}$ \\
\hline Revivum 18-04 & 2 & $\mathrm{I}$ & 0 & $\mathrm{R}$ & $0 ; \mathrm{nc}$ & $\mathrm{R}$ & 8 & $\mathrm{~S}$ & 6 & $\mathrm{~S}$ & $2^{+} \mathrm{c}$ & I & 2 & $\mathrm{I}$ & $2^{-} \mathrm{c}$ & $\mathrm{I}$ & $3^{-} \mathrm{c} / 2^{+}$ & $\mathrm{S} / \mathrm{I}$ \\
\hline Revivum 18-26 & 0 & $\mathrm{R}$ & 0 & $\mathrm{R}$ & $0 ; \mathrm{nc}$ & $\mathrm{R}$ & 7 & $\mathrm{~S}$ & 7 & $\mathrm{~S}$ & $2 c$ & $\mathrm{I}$ & $3^{-}$ & $\mathrm{S}$ & $3^{-} \mathrm{c}$ & $\mathrm{S}$ & $3^{-} \mathrm{C}$ & $\mathrm{S}$ \\
\hline Yeroham 19-03 & 0 & $\mathrm{R}$ & 3 & $\mathrm{~S}$ & $3^{-} \mathrm{C}$ & $\mathrm{S}$ & 4 & $\mathrm{I}$ & 3 & $\mathrm{R}$ & 3 & $\mathrm{~S}$ & 3 & $\mathrm{~S}$ & 3 & $\mathrm{~S}$ & 3 & $\mathrm{~S}$ \\
\hline Yeroham 19-32 & 0 & $\mathrm{R}$ & 2 & $\mathrm{I}$ & $3 / 1 n$ & S/R & 2 & $\mathrm{R}$ & 5 & I & $2-/ 3$ & $\mathrm{I} / \mathrm{S}$ & $3^{-}$ & $\mathrm{S}$ & 3 & $\mathrm{~S}$ & 3 & $\mathrm{~S}$ \\
\hline Sede Boqer 20-22 & 0 & $\mathrm{R}$ & 3 & $\mathrm{~S}$ & $1 \mathrm{n} / 3$ & $\mathrm{R} / \mathrm{S}$ & 2 & $\mathrm{R}$ & 6 & $\mathrm{~S}$ & $2^{-}$ & I & $3^{-}$ & $\mathrm{S}$ & $3^{-}$ & $\mathrm{S}$ & 3 & $\mathrm{~S}$ \\
\hline Sede Boqer $20-45$ & 0 & $\mathrm{R}$ & 3 & $\mathrm{~S}$ & $0 ; \mathrm{n}$ & $\mathrm{R}$ & 7 & $\mathrm{~S}$ & 6 & $\mathrm{~S}$ & $3^{-}$ & $\mathrm{S}$ & 3 & $\mathrm{~S}$ & 3 & $\mathrm{~S}$ & $3^{+}$ & $\mathrm{S}$ \\
\hline Bet Shean 21-15 & 0 & $\mathrm{R}$ & 1 & $\mathrm{R}$ & $0 ; \mathrm{nc}$ & $\mathrm{R}$ & 4 & $\mathrm{I}$ & 7 & $\mathrm{~S}$ & 3 & $\mathrm{~S}$ & $3^{-}$ & $\mathrm{S}$ & 3 & $\mathrm{~S}$ & 3 & $\mathrm{~S}$ \\
\hline Bet Shean 21-46 & 0 & $\mathrm{R}$ & 2 & I & $0 ; n$ & $\mathrm{R}$ & 1 & $\mathrm{R}$ & 2 & $\mathrm{R}$ & $3^{-}$ & $\mathrm{S}$ & 3 & $\mathrm{~S}$ & 3 & $\mathrm{~S}$ & 3 & $\mathrm{~S}$ \\
\hline Mehola 22-16 & 2 & $\mathrm{I}$ & 3 & $\mathrm{~S}$ & $0 ; \mathrm{n} / 2^{-} \mathrm{c}$ & $\mathrm{R} / \mathrm{I}$ & 7 & $\mathrm{~S}$ & 6 & $\mathrm{~S}$ & 3 & $\mathrm{~S}$ & 3 & $\mathrm{~S}$ & 3 & $\mathrm{~S}$ & 3 & $\mathrm{~S}$ \\
\hline Mehola 22-42 & 0 & $\mathrm{R}$ & 3 & $\mathrm{~S}$ & $1^{+} \mathrm{cn}$ & $\mathrm{R}$ & 3 & $\mathrm{R}$ & 2 & $\mathrm{R}$ & 3 & $\mathrm{~S}$ & 3 & $\mathrm{~S}$ & 3 & $\mathrm{~S}$ & 3 & $\mathrm{~S}$ \\
\hline Wadi Qilt 23-67 & 0 & $\mathrm{R}$ & 0 & $\mathrm{R}$ & $2^{+} \mathrm{c}$ & I & 2 & $\mathrm{R}$ & 6 & $\mathrm{~S}$ & 3 & $\mathrm{~S}$ & 3 & $\mathrm{~S}$ & $3 c$ & $\mathrm{~S}$ & 3 & $\mathrm{~S}$ \\
\hline Wadi Qilt 23-72 & 0 & $\mathrm{R}$ & 0 & $\mathrm{R}$ & $3-c$ & $\mathrm{~S}$ & 2 & $\mathrm{R}$ & 5 & $\mathrm{I}$ & $3^{-}$ & $S$ & $3^{-}$ & $\mathrm{S}$ & $3^{-}$ & $\mathrm{S}$ & 3 & $\mathrm{~S}$ \\
\hline Akhziv 24-39 & 0 & $\mathrm{R}$ & 4 & $\mathrm{~S}$ & $0 ; n$ & $\mathrm{R}$ & 2 & $\mathrm{R}$ & 3 & $\mathrm{R}$ & $3^{-} \mathrm{c}$ & $\mathrm{S}$ & 3 & $\mathrm{~S}$ & $3 c$ & $\mathrm{~S}$ & 3 & $\mathrm{~S}$ \\
\hline Akhziv 24-57 & 0 & $\mathrm{R}$ & 4 & $\mathrm{~S}$ & $0 ; \mathrm{nc}$ & $\mathrm{R}$ & 2 & $\mathrm{R}$ & 3 & $\mathrm{R}$ & $3^{-}$ & $\mathrm{S}$ & 3 & $\mathrm{~S}$ & 3 & $\mathrm{~S}$ & 3 & $\mathrm{~S}$ \\
\hline Atlit 25-08 & 0 & $\mathrm{R}$ & 0 & $\mathrm{R}$ & $1 \mathrm{~N}$ & $\mathrm{R}$ & 7 & $\mathrm{~S}$ & 6 & $\mathrm{~S}$ & 3 & $\mathrm{~S}$ & $3^{-}$ & $\mathrm{S}$ & 3 & $\mathrm{~S}$ & 3 & $\mathrm{~S}$ \\
\hline Atlit $25-20$ & 0 & $\mathrm{R}$ & 0 & $\mathrm{R}$ & $1 \mathrm{~N}$ & $\mathrm{R}$ & 8 & $\mathrm{~S}$ & 6 & $\mathrm{~S}$ & 2 & I & $3^{-}$ & $\mathrm{S}$ & 3 & $\mathrm{~S}$ & 3 & $\mathrm{~S}$ \\
\hline Atlit $25-36$ & 1 & $\mathrm{R}$ & 0 & $\mathrm{R}$ & $0 ; \mathrm{n}$ & $\mathrm{R}$ & 2 & $\mathrm{R}$ & 6 & $\mathrm{~S}$ & 3 & $\mathrm{~S}$ & $3^{-}$ & $\mathrm{S}$ & $3^{-}$ & $\mathrm{S}$ & 3 & $\mathrm{~S}$ \\
\hline Caesarea 26-22 & 0 & $\mathrm{R}$ & 0 & $\mathrm{R}$ & $1 \mathrm{~N}$ & $\mathrm{R}$ & 5 & I & 5 & I & $3^{-}$ & $\mathrm{S}$ & $3^{-}$ & $\mathrm{S}$ & 3 & $\mathrm{~S}$ & 3 & $\mathrm{~S}$ \\
\hline Caesarea 26-47 & 0 & $\mathrm{R}$ & 0 & $\mathrm{R}$ & $1 \mathrm{~N}$ & $\mathrm{R}$ & 5 & $\mathrm{I}$ & 6 & $\mathrm{~S}$ & $3^{-}$ & $\mathrm{S}$ & 3 & $\mathrm{~S}$ & $3 \mathrm{c}$ & $\mathrm{S}$ & 3 & $\mathrm{~S}$ \\
\hline Herzliyya 27-55 & 0 & $\mathrm{R}$ & 1 & $\mathrm{R}$ & $2 c$ & I & 2 & $\mathrm{R}$ & 6 & $\mathrm{~S}$ & 3 & $\mathrm{~S}$ & 3 & $\mathrm{~S}$ & 3 & $\mathrm{~S}$ & 3 & $\mathrm{~S}$ \\
\hline Herzliyya 27-59 & 0 & $\mathrm{R}$ & 1 & $\mathrm{R}$ & $1^{+} \mathrm{cn}$ & $\mathrm{R}$ & 1 & $\mathrm{R}$ & 6 & $\mathrm{~S}$ & 3 & $\mathrm{~S}$ & 3 & $\mathrm{~S}$ & 3 & $\mathrm{~S}$ & 3 & $\mathrm{~S}$ \\
\hline Ashqelon 28-03 & 0 & $\mathrm{R}$ & 2 & $\mathrm{I}$ & $0 ; \mathrm{n}$ & $\mathrm{R}$ & 3 & $\mathrm{R}$ & 7 & $\mathrm{~S}$ & $3^{-}$ & $\mathrm{S}$ & 3 & $\mathrm{~S}$ & 3 & $\mathrm{~S}$ & 3 & $\mathrm{~S}$ \\
\hline Ashqelon 28-37 & 0 & $\mathrm{R}$ & 2 & $\mathrm{I}$ & $2 c$ & I & 2 & $\mathrm{R}$ & 3 & $\mathrm{R}$ & 3 & $\mathrm{~S}$ & 3 & $\mathrm{~S}$ & 3 & $\mathrm{~S}$ & 3 & $\mathrm{~S}$ \\
\hline Machtesh 31-13 & 2 & $\mathrm{I}$ & 0 & $\mathrm{R}$ & $2-/ 3$ & $\mathrm{I} / \mathrm{S}$ & 2 & $\mathrm{R}$ & 5 & $\mathrm{I}$ & $3^{-}$ & $\mathrm{S}$ & 3 & $\mathrm{~S}$ & $3^{-}$ & $\mathrm{S}$ & 3 & $\mathrm{~S}$ \\
\hline Machtesh 31-22 & 0 & $\mathrm{R}$ & 0 & $\mathrm{R}$ & $2^{-} \mathrm{c}$ & I & 3 & $\mathrm{R}$ & 6 & $\mathrm{~S}$ & $2^{+} \mathrm{c}$ & $\mathrm{I}$ & 3 & $\mathrm{~S}$ & $3^{-}$ & $\mathrm{S}$ & 3 & $\mathrm{~S}$ \\
\hline Ein Zukim 32-05 & 2 & I & $0 / 3$ & $\mathrm{R} / \mathrm{S}$ & 0 & $\mathrm{R}$ & 3 & $\mathrm{R}$ & 6 & $\mathrm{~S}$ & $3^{-}$ & $\mathrm{S}$ & 3 & $\mathrm{~S}$ & 3 & $\mathrm{~S}$ & 3 & $\mathrm{~S}$ \\
\hline Ein Zukim 32-11 & 0 & $\mathrm{R}$ & 3 & $\mathrm{~S}$ & 0 & $\mathrm{R}$ & 6 & $\mathrm{~S}$ & 6 & $\mathrm{~S}$ & $3^{-}$ & $\mathrm{S}$ & 3 & $\mathrm{~S}$ & $3^{-}$ & $\mathrm{S}$ & $3^{-}$ & $\mathrm{S}$ \\
\hline Avedat $33-28$ & 0 & $\mathrm{R}$ & 3 & $\mathrm{~S}$ & $2^{+} \mathrm{c}$ & I & 3 & $\mathrm{R}$ & 2 & $\mathrm{R}$ & $1^{+}$ & $\mathrm{R}$ & 3 & $\mathrm{~S}$ & $3^{-}$ & $\mathrm{S}$ & 3 & $\mathrm{~S}$ \\
\hline Avedat 33-54 & 0 & $\mathrm{R}$ & 0 & $\mathrm{R}$ & $3^{-}$ & $\mathrm{S}$ & 5 & $\mathrm{I}$ & 7 & $\mathrm{~S}$ & $3^{-}$ & $\mathrm{S}$ & $3^{-}$ & $\mathrm{S}$ & $3^{-}$ & $\mathrm{S}$ & 3 & $\mathrm{~S}$ \\
\hline Number/percent of: & $\mathrm{N}$ & $\%$ & $\mathrm{~N}$ & $\%$ & $\mathrm{~N}$ & $\%$ & $\mathrm{~N}$ & $\%$ & $\mathrm{~N}$ & $\%$ & $\mathrm{~N}$ & $\%$ & $\mathrm{~N}$ & $\%$ & $\mathrm{~N}$ & $\%$ & $\mathrm{~N}$ & $\%$ \\
\hline Low IR/IT & 57 & 90 & 32 & 51 & 42 & 67 & 33 & 52 & 11 & 17 & 8 & 13 & 1 & 2 & 2 & 3 & 0 & 0 \\
\hline Intermediate IR/IT & 5 & 8 & 12 & 19 & 15 & 23 & 10 & 16 & 18 & 29 & 8 & 13 & 3 & 5 & 5 & 8 & 0 & 0 \\
\hline High IR/IT & 1 & 2 & 19 & 30 & 6 & 10 & 20 & 32 & 34 & 54 & 47 & 74 & 59 & 93 & 56 & 89 & 63 & 100 \\
\hline
\end{tabular}

a Accessions exhibiting infection responses of 0 to 1,2 , and 3 to 5 were considered resistant, intermediate, and susceptible, respectively, to $S$. passerinii (Toubia-Rahme and Steffenson, 1999 [45]).

b Accessions exhibiting infection types of 0 to 1, 2, and 3 to 4 were considered resistant, intermediate, and susceptible, respectively, to B. graminis f. sp. hordei (Torp et al., 1978 [44]).

c Accessions exhibiting infection types of 0 to 1, 2, and 3 to 4 were considered resistant, intermediate, and susceptible, respectively, to Puccinia hordei (Levine and Cherewick, 1952 [24]) and P. graminis f. sp. tritici (Miller and Lambert, 1955 [29]).

d Accessions exhibiting infection responses of 1 to 3, 4 to 5, and 6 to 9 were considered resistant, intermediate, and susceptible, respectively, to Pyrenophora teres f. teres (Tekauz 1985 [43]) and Cochliobolus sativus (Fetch and Steffenson, 1999 [9]).

e IP = infection phenotypes, where $\mathrm{R}=$ resistant, $\mathrm{I}=$ intermediate resistant, and $\mathrm{S}=$ susceptible. 
Table 5. Disease reactions of Hordeum spontaneum accessions sampled from Jordan to Septoria passerinii, Blumeria graminis f. sp. hordei, Puccinia hordei, Pyrenophora teres f. teres, Cochliobolus sativus, and Puccinia graminis f. sp. tritici

\begin{tabular}{|c|c|c|c|c|c|c|c|c|c|c|c|c|c|c|c|c|c|c|}
\hline \multirow[b]{3}{*}{ Line } & \multirow{2}{*}{\multicolumn{2}{|c|}{$\begin{array}{c}\text { Septoria } \\
\text { passerinii }^{\mathrm{a}}\end{array}$}} & \multirow{2}{*}{\multicolumn{2}{|c|}{$\begin{array}{c}\text { B. g. f. sp. } \\
\text { hordei }^{\mathrm{b}}\end{array}$}} & \multirow{2}{*}{\multicolumn{2}{|c|}{$\begin{array}{c}\text { Puccinia } \\
\text { hordei }^{\mathrm{c}}\end{array}$}} & \multirow{2}{*}{\multicolumn{2}{|c|}{$\begin{array}{l}\text { Pyrenophora } \\
\text { teres f. teres }{ }^{\mathrm{d}}\end{array}$}} & \multirow{2}{*}{\multicolumn{2}{|c|}{$\begin{array}{c}\text { Cochliobolus } \\
\text { sativus }^{\mathrm{d}}\end{array}$}} & \multicolumn{8}{|c|}{ Puccinia graminis f. sp. tritici } \\
\hline & & & & & & & & & & & \multicolumn{2}{|c|}{$\operatorname{MCCF} l^{c}$} & \multicolumn{2}{|c|}{ MCCF hi ${ }^{\mathrm{c}}$} & \multicolumn{2}{|c|}{ QCCJ loc } & QC & $\mathbf{J} \mathbf{h i}^{\mathbf{c}}$ \\
\hline & IR & $\mathbf{I P}^{\mathbf{e}}$ & IR & IP & IT & IP & IR & IP & IR & IP & IT & IP & IT & IP & IT & IP & IT & IP \\
\hline Shūna, N. 1-32 & 0 & $\mathrm{R}$ & 0 & $\mathrm{R}$ & $1^{+} \mathrm{c}$ & $\mathrm{R}$ & 2 & $\mathrm{R}$ & 5 & I & 3 & $\mathrm{~S}$ & $3^{+}$ & $\mathrm{S}$ & 3 & S & 3 & S \\
\hline Shūna, N. 1-43 & 4 & $\mathrm{~S}$ & 0 & $\mathrm{R}$ & $1^{+} \mathrm{cn}$ & $\mathrm{R}$ & 2 & $\mathrm{R}$ & 2 & $\mathrm{R}$ & 3 & $\mathrm{~S}$ & 3 & $\mathrm{~S}$ & 3 & $S$ & 3 & $S$ \\
\hline Irbid 2-14 & 0 & $\mathrm{R}$ & 3 & $\mathrm{~S}$ & $0 ; \mathrm{n}$ & $\mathrm{R}$ & 3 & $\mathrm{R}$ & 2 & $\mathrm{R}$ & 3 & $\mathrm{~S}$ & $3^{-}$ & $\mathrm{S}$ & 3 & $\mathrm{~S}$ & 3 & $\mathrm{~S}$ \\
\hline Irbid 2-34 & 1 & $\mathrm{R}$ & 1 & $\mathrm{R}$ & 3 & $\mathrm{~S}$ & 7 & $\mathrm{~S}$ & 3 & $\mathrm{R}$ & 3 & $\mathrm{~S}$ & $3^{-}$ & S & 3 & S & 3 & $S$ \\
\hline Irbid, E. 3-31 & 0 & $\mathrm{R}$ & 3 & $\mathrm{~S}$ & $1^{+} \mathrm{N}$ & $\mathrm{R}$ & 4 & I & 6 & $\mathrm{~S}$ & $3^{-}$ & $\mathrm{S}$ & $3^{-}$ & S & $3^{-}$ & S & $3^{+}$ & S \\
\hline Irbid, E. 3-36 & 0 & $\mathrm{R}$ & 3 & S & 3 & S & 2 & $\mathrm{R}$ & 6 & S & $3^{-}$ & $\mathrm{S}$ & $3^{+}$ & S & $3^{-} \mathrm{c}$ & S & 3 & S \\
\hline Mafraq, W. 4-07 & 0 & $\mathrm{R}$ & 2 & I & $3^{-}$ & S & 2 & $\mathrm{R}$ & 5 & I & 3 & $\mathrm{~S}$ & $3^{-}$ & $\mathrm{S}$ & 3 & S & 3 & S \\
\hline Mafraq, W. 4-11 & 0 & $\mathrm{R}$ & 3 & $S$ & $3 \mathrm{cn}$ & S & 5 & I & 5 & I & $3^{-}$ & $S$ & 3 & $\mathrm{~S}$ & $3^{-}$ & S & $3 \mathrm{C}$ & S \\
\hline Mafraq 5-34 & 0 & $\mathrm{R}$ & 3 & $\mathrm{~S}$ & 3 & $\mathrm{~S}$ & 4 & I & 6 & $\mathrm{~S}$ & 3 & $\mathrm{~S}$ & $3^{-}$ & $\mathrm{S}$ & 3 & $\mathrm{~S}$ & 3 & $\mathrm{~S}$ \\
\hline Zarqa 6-21 & 1 & $\mathrm{R}$ & 2 & I & $1^{+} \mathrm{c}$ & $\mathrm{R}$ & 2 & $\mathrm{R}$ & 5 & I & $2^{-}$ & I & 3 & $\mathrm{~S}$ & $2 \mathrm{c}$ & I & $3^{-} \mathrm{c}$ & $\mathrm{S}$ \\
\hline Zarqa 6-38 & 2 & I & 1 & $\mathrm{R}$ & $2^{+}$ & I & 3 & $\mathrm{R}$ & 8 & $\mathrm{~S}$ & 3 & $\mathrm{~S}$ & 3 & $\mathrm{~S}$ & 3 & $\mathrm{~S}$ & 3 & $\mathrm{~S}$ \\
\hline Amman 7-06 & 0 & $\mathrm{R}$ & 0 & $\mathrm{R}$ & $2 \mathrm{c}$ & I & 4 & I & 3 & $\mathrm{R}$ & $3^{-}$ & $\mathrm{S}$ & 3 & $\mathrm{~S}$ & 3 & $\mathrm{~S}$ & $3^{-}$ & $\mathrm{S}$ \\
\hline Amman 7-48 & 1 & $\mathrm{R}$ & 0 & $\mathrm{R}$ & $3 c$ & S & 6 & $\mathrm{~S}$ & 4 & I & $3^{-}$ & $\mathrm{S}$ & 3 & $\mathrm{~S}$ & $3^{+}$ & S & $3 \mathrm{C}$ & S \\
\hline Madaba 8-08 & 0 & $\mathrm{R}$ & 0 & $\mathrm{R}$ & $1^{+} \mathrm{c}$ & $\mathrm{R}$ & 7 & $\mathrm{~S}$ & 3 & $\mathrm{R}$ & $3^{-}$ & $\mathrm{S}$ & $3^{-}$ & $\mathrm{S}$ & $3^{-}$ & S & 3 & S \\
\hline Madaba 8-32 & 1 & $\mathrm{R}$ & 4 & $\mathrm{~S}$ & 1n & $\mathrm{R}$ & 3 & $\mathrm{R}$ & 3 & $\mathrm{R}$ & $3^{-}$ & $S$ & $3^{-}$ & $\mathrm{S}$ & $2^{-}$ & I & $3^{-}$ & S \\
\hline Mt. Nebo 9-25 & 1 & $\mathrm{R}$ & 0 & $\mathrm{R}$ & $2 \mathrm{C}$ & I & 7 & $\mathrm{~S}$ & 8 & $\mathrm{~S}$ & 3 & $\mathrm{~S}$ & $3^{-}$ & $\mathrm{S}$ & $3^{-}$ & $\mathrm{S}$ & $3^{-}$ & $\mathrm{S}$ \\
\hline Mt. Nebo 9-39 & 0 & $\mathrm{R}$ & 3 & $\mathrm{~S}$ & $3^{-}$ & $\mathrm{S}$ & 3 & $\mathrm{R}$ & 6 & $\mathrm{~S}$ & $3^{-}$ & $\mathrm{S}$ & $3^{-}$ & $\mathrm{S}$ & $3^{-}$ & $\mathrm{S}$ & $3^{-}$ & $\mathrm{S}$ \\
\hline Wadi Wala $10-05$ & 0 & $\mathrm{R}$ & 0 & $\mathrm{R}$ & $2^{-} \mathrm{c}$ & I & 2 & $\mathrm{R}$ & 6 & $\mathrm{~S}$ & $3^{-}$ & $\mathrm{S}$ & 3 & $\mathrm{~S}$ & $2^{+} \mathrm{c}$ & I & $3^{-}$ & $\mathrm{S}$ \\
\hline Wadi Wala 10-38 & 0 & $\mathrm{R}$ & 2 & $\mathrm{I}$ & $2 \mathrm{c}$ & I & 3 & $\mathrm{R}$ & 4 & I & 3 & $\mathrm{~S}$ & 3 & $\mathrm{~S}$ & 3 & S & 3 & $S$ \\
\hline Wadi Mūjib 11-09 & 4 & $\mathrm{~S}$ & 3 & $\mathrm{~S}$ & $3^{-} \mathrm{C}$ & $\mathrm{S}$ & 5 & I & 6 & $\mathrm{~S}$ & 3 & $\mathrm{~S}$ & 3 & $\mathrm{~S}$ & 3 & $\mathrm{~S}$ & $3 \mathrm{C}$ & $\mathrm{S}$ \\
\hline Wadi Mūjib 11-17 & 1 & $\mathrm{R}$ & 3 & $\mathrm{~S}$ & $2^{+} \mathrm{c}$ & I & 3 & $\mathrm{R}$ & 6 & S & 3 & $\mathrm{~S}$ & 3 & $\mathrm{~S}$ & 3 & S & 3 & S \\
\hline Talal 12-13 & 5 & $\mathrm{~S}$ & 0 & $\mathrm{R}$ & 3 & S & 7 & $\mathrm{~S}$ & 6 & S & $3^{-}$ & $\mathrm{S}$ & $3^{-}$ & $\mathrm{S}$ & $3^{-}$ & S & $3 \mathrm{C}$ & S \\
\hline Talal 12-21 & 2 & I & 4 & $\mathrm{~S}$ & $2 \mathrm{c}$ & I & 2 & $\mathrm{R}$ & 4 & I & 3 & $\mathrm{~S}$ & $3^{-}$ & $\mathrm{S}$ & 3 & $\mathrm{~S}$ & $3 \mathrm{C}$ & $\mathrm{S}$ \\
\hline Karak 13-10 & 3 & $\mathrm{~S}$ & 3 & $\mathrm{~S}$ & $0 ; \mathrm{N}$ & $\mathrm{R}$ & 7 & $\mathrm{~S}$ & 4 & I & 3 & $\mathrm{~S}$ & $3^{-}$ & $\mathrm{S}$ & 3 & S & 3 & S \\
\hline Karak 13-43 & 0 & $\mathrm{R}$ & 3 & $\mathrm{~S}$ & $1 \mathrm{n}$ & $\mathrm{R}$ & 2 & $\mathrm{R}$ & 4 & I & 3 & $\mathrm{~S}$ & 3 & $\mathrm{~S}$ & 3 & S & 3 & S \\
\hline Wadi Hasa 14-02 & 0 & $\mathrm{R}$ & 2 & I & $0 ; \mathrm{n}$ & $\mathrm{R}$ & 4 & I & 6 & $\mathrm{~S}$ & 3 & $\mathrm{~S}$ & $3^{-}$ & $\mathrm{S}$ & $3^{-}$ & $\mathrm{S}$ & 3 & $\mathrm{~S}$ \\
\hline Wadi Hasa 14-27 & 0 & $\mathrm{R}$ & 1 & $\mathrm{R}$ & $1 \mathrm{nc}$ & $\mathrm{R}$ & 2 & $\mathrm{R}$ & 6 & $\mathrm{~S}$ & 3 & $\mathrm{~S}$ & 3 & $\mathrm{~S}$ & $3^{-} \mathrm{c}$ & $\mathrm{S}$ & $3 \mathrm{C}$ & $\mathrm{S}$ \\
\hline Laban 15-09 & 5 & $\mathrm{~S}$ & 1 & $\mathrm{R}$ & $0 ; \mathrm{N}$ & $\mathrm{R}$ & $3 / 6$ & $\mathrm{R} / \mathrm{S}$ & 6 & $\mathrm{~S}$ & $3^{-}$ & $\mathrm{S}$ & $3^{-}$ & $\mathrm{S}$ & $3^{-}$ & $\mathrm{S}$ & 3 & $\mathrm{~S}$ \\
\hline Laban 15-39 & 0 & $\mathrm{R}$ & 0 & $\mathrm{R}$ & $3-\mathrm{c}$ & S & 8 & $\mathrm{~S}$ & 7 & S & $3^{-}$ & $\mathrm{S}$ & $3^{-}$ & S & $3^{+}$ & $\mathrm{S}$ & 3 & S \\
\hline Tafila 16-04 & 3 & $S$ & 0 & $\mathrm{R}$ & $3^{-}$ & S & 7 & $\mathrm{~S}$ & 7 & S & 3 & $\mathrm{~S}$ & 3 & S & 3 & $\mathrm{~S}$ & 3 & S \\
\hline Tafila $16-33$ & 2 & I & 2 & $\mathrm{I}$ & $2 \mathrm{c}$ & I & 3 & $\mathrm{R}$ & 5 & $\mathrm{I}$ & 3 & $\mathrm{~S}$ & 3 & S & 3 & $S$ & 3 & S \\
\hline Danā 17-43 & 5 & $\mathrm{~S}$ & 3 & $\mathrm{~S}$ & $3^{-} \mathrm{c}$ & $\mathrm{S}$ & 7 & $\mathrm{~S}$ & 3 & $\mathrm{R}$ & 3 & $\mathrm{~S}$ & 3 & $\mathrm{~S}$ & $3^{+}$ & $\mathrm{S}$ & 3 & $\mathrm{~S}$ \\
\hline Danā 17-48 & 5 & $\mathrm{~S}$ & 3 & $\mathrm{~S}$ & 3 & $\mathrm{~S}$ & $5 / 7$ & $\mathrm{I} / \mathrm{S}$ & 5 & I & 3 & $\mathrm{~S}$ & 3 & $\mathrm{~S}$ & 3 & $\mathrm{~S}$ & 3 & $\mathrm{~S}$ \\
\hline Wadi Mūsā 18-08 & 5 & $\mathrm{~S}$ & 4 & $\mathrm{~S}$ & $2^{+} \mathrm{c}$ & I & 4 & I & 7 & S & 3 & $\mathrm{~S}$ & 3 & $\mathrm{~S}$ & $3^{+}$ & $\mathrm{S}$ & 3 & S \\
\hline Wadi Mūsā 18-42 & 5 & $\mathrm{~S}$ & 3 & $\mathrm{~S}$ & $3^{-} \mathrm{c}$ & $\mathrm{S}$ & 6 & $\mathrm{~S}$ & 5 & I & $3^{-}$ & $\mathrm{S}$ & 3 & $\mathrm{~S}$ & 3 & $\mathrm{~S}$ & 3 & $\mathrm{~S}$ \\
\hline Safi 19-23 & 0 & $\mathrm{R}$ & 3 & $\mathrm{~S}$ & $3^{-}$ & $\mathrm{S}$ & 7 & $\mathrm{~S}$ & 5 & I & $3^{+}$ & $\mathrm{S}$ & 3 & $\mathrm{~S}$ & 3 & $\mathrm{~S}$ & 3 & S \\
\hline Safi $19-29$ & 0 & $\mathrm{R}$ & 2 & $\mathrm{I}$ & $2 \mathrm{c}$ & I & 2 & $\mathrm{R}$ & 6 & $\mathrm{~S}$ & 3 & $\mathrm{~S}$ & 3 & $\mathrm{~S}$ & 3 & S & 3 & S \\
\hline Shūna S. 20-04 & 5 & $\mathrm{~S}$ & 0 & $\mathrm{R}$ & $3^{-} \mathrm{c}$ & $\mathrm{S}$ & 7 & $\mathrm{~S}$ & 6 & $\mathrm{~S}$ & 3 & $S$ & 3 & $\mathrm{~S}$ & $3^{-}$ & S & 3 & $S$ \\
\hline Shūna S. 20-14 & 0 & $\mathrm{R}$ & 0 & $\mathrm{R}$ & $2 \mathrm{C}$ & I & 2 & $\mathrm{R}$ & 4 & I & 3 & $\mathrm{~S}$ & 3 & $\mathrm{~S}$ & $3^{+}$ & S & 3 & S \\
\hline 'Ira 21-21 & 0 & $\mathrm{R}$ & 3 & $\mathrm{~S}$ & $1^{+} \mathrm{C}$ & $\mathrm{R}$ & 3 & $\mathrm{R}$ & 4 & I & 3 & $\mathrm{~S}$ & 3 & $\mathrm{~S}$ & $3^{+}$ & S & 3 & S \\
\hline 'Ira 21-33 & 0 & $\mathrm{R}$ & 3 & $\mathrm{~S}$ & $2 \mathrm{C}$ & I & 2 & $\mathrm{R}$ & 4 & I & 3 & $\mathrm{~S}$ & $3^{+}$ & $\mathrm{S}$ & $3^{+}$ & S & 3 & S \\
\hline Salt 22-07 & 0 & $\mathrm{R}$ & 2 & I & $0 ; \mathrm{c}$ & $\mathrm{R}$ & 2 & $\mathrm{R}$ & 5 & I & $3^{-} \mathrm{c}$ & $\mathrm{S}$ & $3^{-}$ & $\mathrm{S}$ & $3^{-}$ & $\mathrm{S}$ & 3 & $\mathrm{~S}$ \\
\hline Salt 22-15 & 0 & $\mathrm{R}$ & 3 & $\mathrm{~S}$ & $2 \mathrm{C}$ & I & 6 & $\mathrm{~S}$ & 6 & $\mathrm{~S}$ & 3 & $\mathrm{~S}$ & 3 & $\mathrm{~S}$ & $3^{-} \mathrm{c}$ & $\mathrm{S}$ & 3 & $\mathrm{~S}$ \\
\hline Jarash 23-06 & 0 & $\mathrm{R}$ & 0 & $\mathrm{R}$ & $0 ; \mathrm{N}$ & $\mathrm{R}$ & 4 & I & 5 & I & 3 & $\mathrm{~S}$ & 3 & $\mathrm{~S}$ & 3 & S & $3^{-}$ & S \\
\hline Jarash 23-44 & 4 & S & 0 & $\mathrm{R}$ & $1^{+} \mathrm{c}$ & $\mathrm{R}$ & 2 & $\mathrm{R}$ & 4 & I & 3 & $\mathrm{~S}$ & 3 & $\mathrm{~S}$ & $3^{+}$ & S & 3 & S \\
\hline Sakib 24-25 & 0 & $\mathrm{R}$ & 0 & $\mathrm{R}$ & $2 \mathrm{c}$ & I & 6 & $\mathrm{~S}$ & 6 & S & 3 & $S$ & 3 & $\mathrm{~S}$ & $3^{-}$ & $\mathrm{S}$ & 3 & S \\
\hline Sakib 24-32 & 0 & $\mathrm{R}$ & 3 & $\mathrm{~S}$ & $1 \mathrm{cn}$ & $\mathrm{R}$ & 3 & $\mathrm{R}$ & 6 & $\mathrm{~S}$ & 3 & $\mathrm{~S}$ & $3^{-}$ & $\mathrm{S}$ & 3 & $\mathrm{~S}$ & 3 & $\mathrm{~S}$ \\
\hline Ajlun 25-29 & 0 & $\mathrm{R}$ & 0 & $\mathrm{R}$ & $1 \mathrm{nc}$ & $\mathrm{R}$ & 5 & I & 7 & $\mathrm{~S}$ & $3^{-}$ & $\mathrm{S}$ & $3^{-}$ & $\mathrm{S}$ & 3 & $\mathrm{~S}$ & 3 & S \\
\hline Ajlun 25-35 & 0 & $\mathrm{R}$ & 0 & $\mathrm{R}$ & $0 ; \mathrm{n}$ & $\mathrm{R}$ & $5 / 7$ & $\mathrm{I} / \mathrm{S}$ & 7 & $\mathrm{~S}$ & $3^{-}$ & $S$ & $3^{-}$ & $\mathrm{S}$ & 3 & $\mathrm{~S}$ & 3 & S \\
\hline Irbid, S. 26-09 & 2 & I & 0 & $\mathrm{R}$ & $1 \mathrm{~N}$ & $\mathrm{R}$ & 2 & $\mathrm{R}$ & 4 & I & 3 & $\mathrm{~S}$ & $3^{+}$ & $\mathrm{S}$ & 3 & $\mathrm{~S}$ & 3 & $S$ \\
\hline Irbid, S. 26-26 & 0 & $\mathrm{R}$ & 3 & $\mathrm{~S}$ & $2 \mathrm{cn}$ & I & 2 & $\mathrm{R}$ & 7 & $\mathrm{~S}$ & 3 & $\mathrm{~S}$ & $3^{+}$ & $\mathrm{S}$ & 3 & S & 3 & S \\
\hline El Hamma 27-01 & 0 & $\mathrm{R}$ & 2 & I & $1^{+} \mathrm{c}$ & $\mathrm{R}$ & 5 & I & 6 & $\mathrm{~S}$ & 3 & $\mathrm{~S}$ & $3^{-}$ & $\mathrm{S}$ & 3 & S & 3 & S \\
\hline El Hamma 27-28 & 1 & $\mathrm{R}$ & 0 & $\mathrm{R}$ & $1^{+} \mathrm{c}$ & $\mathrm{R}$ & 7 & $\mathrm{~S}$ & 5 & I & 3 & $\mathrm{~S}$ & $3^{+}$ & $\mathrm{S}$ & $3^{+}$ & $\mathrm{S}$ & 3 & $\mathrm{~S}$ \\
\hline Number/percent of: & $\mathrm{N}$ & $\%$ & $\mathrm{~N}$ & $\%$ & $\mathrm{~N}$ & $\%$ & $\mathrm{~N}$ & $\%$ & $\mathrm{~N}$ & $\%$ & $\mathrm{~N}$ & $\%$ & $\mathrm{~N}$ & $\%$ & $\mathrm{~N}$ & $\%$ & $\mathrm{~N}$ & $\%$ \\
\hline Low IR/ITs & 37 & 70 & 23 & 43 & 22 & 42 & 26 & 49 & 7 & 13 & 0 & 0 & 0 & 0 & 0 & 0 & 0 & 0 \\
\hline Intermediate IR/ITs & 4 & 7 & 8 & 15 & 15 & 28 & 12 & 23 & 21 & 40 & 1 & 2 & 0 & 0 & 3 & 6 & 0 & 0 \\
\hline High IR/ITs & 12 & 23 & 22 & 42 & 16 & 30 & 15 & 28 & 25 & 47 & 52 & 98 & 53 & 100 & 50 & 94 & 53 & 100 \\
\hline
\end{tabular}

a Accessions exhibiting infection responses of 0 to 1,2 , and 3 to 5 were considered resistant, intermediate, and susceptible, respectively, to $S$. passerinii (Toubia-Rahme and Steffenson, 1999 [45]).

b Accessions exhibiting infection types of 0 to 1,2, and 3 to 4 were considered resistant, intermediate, and susceptible, respectively, to $B$. graminis f. sp. hordei (Torp et al., 1978 [44]).

${ }^{\mathrm{c}}$ Accessions exhibiting infection types of 0 to 1,2, and 3 to 4 were considered resistant, intermediate, and susceptible, respectively, to Puccinia hordei (Levine and Cherewick, 1952 [24]) and P. graminis f. sp. tritici (Miller and Lambert, 1955 [29]).

${ }^{d}$ Accessions exhibiting infection responses of 1 to 3, 4 to 5, and 6 to 9 were considered resistant, intermediate, and susceptible, respectively, to Pyrenophora teres f. teres (Tekauz 1985 [43]) and Cochliobolus sativus (Fetch and Steffenson, 1999 [9]).

${ }^{\mathrm{e}} \mathrm{IP}=$ infection phenotypes, where $\mathrm{R}=$ resistant, $\mathrm{I}=$ intermediate resistant, and $\mathrm{S}=$ susceptible. 
result confirms previous studies documenting diversity for reaction to different diseases (e.g., powdery mildew, leaf rust, and leaf scald) in this wild species $(1,15,30)$. Other studies reporting a high level of macro-scale diversity in $H$. spontaneum have included assays of DNA polymorphism $(5,6,46)$, allozymic variation in proteins $(33,35)$, growth physiology $(48)$, and agronomic traits (34). The level of macroscale diversity to individual pathogens varied greatly in this study as measured by Shannon's information index. In the combined analysis from both Israel and Jordan, the highest level of diversity was observed for reaction to $B$. graminis f. sp. hordei (1.025) and Pyrenophora teres f. teres (1.021), and the lowest to $S$. passerinii (0.615) and Puccinia graminis f. sp. tritici (0.519). The low level of diversity found for reaction to $S$. passerinii and Puccinia graminis f. sp. tritici was due to a high frequency of resistance and susceptibility, respectively. In addition to macro-scale diversity, we also detected micro-scale diversity within individual collection sites where accessions were gathered no closer than $1 \mathrm{~m}$ apart along transects of 25 to 200 m. Micro-scale diversity within individual collection sites also was found for powdery mildew and leaf rust reaction by Jana and Nevo (15), who studied accessions of $H$. spontaneum from Iran, Syria, and Turkey. Resistant and susceptible plants were identified within individual accessions (originally derived from single plants) to all diseases except SSLB, indicating that genetic heterozygosity exists for many resistance loci. This phenomenon appears to be a common feature of gramineous species at their center of origin (53). The disease evaluations in this investigation were all done on seedling plants to just one or two pathotypes of each pathogen. As such, these evaluations assess the effects of seedling resistance genes with major effects and therefore represent only a small fraction of the potential diversity for disease resistance that may be important in agriculture. A wide spectrum of diversity for adult plant resistance (both qualitative and quantitative) also exists in $H$. spontaneum (3), but was not considered in this investigation. With the high level of macro- and micro-scale diversity for disease reaction, extensive genetic heterozygosity for resis- tance loci, and largely uncharacterized mechanisms of adult plant resistance, $H$. spontaneum is an incredibly rich and largely untapped source of unique disease resistance alleles for cultivated barley improvement.

The environment can be a key component contributing to the range of genetic diversity found in various organisms (49). Patterns of genetic variation in $H$. spontaneum, including disease resistance, are influenced by environmental factors such as temperature and humidity and are not random $(33,36)$. Environmental conditions that favor high disease pressure should increase selection for disease resistance. This implies that possible locations of resistance sources can be predicted using environmental and ecological information. However, in this study, there generally was not a relationship between environment and disease resistance. Low but significant correlations were found between the level of resistance to Puccinia hordei and the amount of rainfall and level of humidity at collection sites in both Israel and Jordan. Higher levels of leaf rust resistance were associated with higher rainfall and humid-

Table 6. Frequency and diversity index of Hordeum spontaneum accessions from Israel and Jordan exhibiting resistant, intermediate, and susceptible reactions to Septoria passerinii, Blumeria graminis f. sp. hordei, Puccinia hordei, Pyrenophora teres f. teres, Cochliobolus sativus, and Puccinia graminis f. sp. tritici

\begin{tabular}{|c|c|c|c|c|c|c|c|c|c|c|c|c|}
\hline \multirow[b]{2}{*}{ Pathogen } & \multicolumn{4}{|c|}{ Israel $(n=63)$} & \multicolumn{4}{|c|}{ Jordan $(n=53)$} & \multicolumn{4}{|c|}{ Combined $(n=116)$} \\
\hline & $\mathbf{R}^{\mathbf{a}}$ & $\mathbf{I}^{\mathbf{a}}$ & $\mathbf{S}^{\mathbf{a}}$ & $\mathbf{h}_{\mathrm{s}}^{\mathbf{b}}$ & $\mathbf{R}^{\mathbf{a}}$ & $\mathbf{I}^{\mathbf{a}}$ & $\mathbf{S}^{\mathbf{a}}$ & $\mathbf{h}_{\mathrm{s}}^{\mathbf{b}}$ & $\mathbf{R}^{\mathbf{a}}$ & $\mathbf{I}^{\mathbf{a}}$ & $\mathbf{S}^{\mathbf{a}}$ & $\mathbf{h}_{\mathrm{s}}^{\mathbf{b}}$ \\
\hline S. passerinii & 0.905 & 0.079 & 0.016 & 0.357 & 0.698 & 0.075 & 0.226 & 0.781 & 0.810 & 0.078 & 0.112 & 0.615 \\
\hline $\begin{array}{l}\text { B. graminis f. sp. } \\
\text { hordei }\end{array}$ & 0.508 & 0.190 & 0.302 & 1.021 & 0.434 & 0.151 & 0.415 & 1.012 & 0.474 & 0.172 & 0.353 & 1.025 \\
\hline Puccinia hordei & 0.667 & 0.238 & 0.095 & 0.836 & 0.415 & 0.283 & 0.302 & 1.084 & 0.552 & 0.259 & 0.190 & 0.993 \\
\hline $\begin{array}{l}\text { Pyrenophora teres } \\
\text { f. teres }\end{array}$ & 0.524 & 0.159 & 0.317 & 0.995 & 0.491 & 0.226 & 0.283 & 1.042 & 0.509 & 0.190 & 0.302 & 1.021 \\
\hline C. sativus & 0.175 & 0.286 & 0.539 & 0.996 & 0.132 & 0.396 & 0.472 & 0.988 & 0.155 & 0.336 & 0.509 & 0.999 \\
\hline $\begin{array}{l}\text { Puccinia graminis } \\
\text { f. sp. triticic }\end{array}$ & 0.127 & 0.127 & 0.746 & 0.743 & 0 & 0.019 & 0.981 & 0.094 & 0.069 & 0.078 & 0.853 & 0.519 \\
\hline
\end{tabular}

${ }^{a}$ Frequency of accessions exhibiting resistant (R), intermediate (I), or susceptible (S) reactions.

${ }^{\mathrm{b}}$ Shannon's diversity index where $h_{s}=-\Sigma p_{i} \ln \left(p_{i}\right)$, with maximum $h_{s}=1.099$.

${ }^{\mathrm{c}}$ Diversity based on reaction to pathotype MCCF (incubated at low temperature) only.

Table 7. Pearson correlation coefficients between environmental parameters and infection response to Septoria passerinii, Blumeria graminis f. sp. hordei, Puccinia hordei, Pyrenophora teres f. teres, Cochliobolus sativus, and Puccinia graminis f. sp. tritici

\begin{tabular}{|c|c|c|c|c|c|}
\hline \multirow[b]{2}{*}{ Pathogen } & \multicolumn{5}{|c|}{ Environmental variables $^{\mathbf{a}}$} \\
\hline & Altitude & TJan & TAug & Rain & Humidity \\
\hline \multicolumn{6}{|l|}{ Israel } \\
\hline S. passerinii & 0.041 & -0.036 & 0.052 & -0.071 & -0.109 \\
\hline B. hordei f. sp. hordei & $-0.252 * \mathrm{~b}$ & 0.162 & $0.267^{*}$ & -0.223 & $-0.282^{*}$ \\
\hline Puccinia hordei & 0.022 & 0.001 & 0.100 & $-0.261^{*}$ & $-0.253^{*}$ \\
\hline Pyrenophora teres f. teres & 0.153 & -0.183 & -0.063 & 0.103 & -0.122 \\
\hline C. sativus & 0.207 & -0.164 & -0.169 & 0.133 & 0.031 \\
\hline Puccinia graminis f. sp. tritici & -0.163 & 0.206 & $0.265^{*}$ & -0.057 & 0.058 \\
\hline \multicolumn{6}{|l|}{ Jordan } \\
\hline S. passerinii & 0.104 & -0.087 & -0.121 & -0.183 & -0.149 \\
\hline B. hordei f. sp. hordei & 0.129 & -0.163 & -0.189 & -0.186 & -0.054 \\
\hline Puccinia hordei & -0.114 & 0.107 & 0.097 & $-0.481^{* *}$ & $-0.301^{*}$ \\
\hline Pyrenophora teres f. teres & 0.108 & -0.002 & 0.035 & -0.051 & -0.065 \\
\hline C. sativus & 0.116 & -0.122 & -0.100 & -0.027 & 0.119 \\
\hline Puccinia graminis f. sp. tritici ${ }^{\mathrm{c}}$ & NA & NA & NA & NA & NA \\
\hline
\end{tabular}

${ }^{\mathrm{a}}$ Environmental variables where Alt $=$ altitude, TJan = mean January temperature, TAug = mean August temperature, Rain = mean annual precipitation, and Hum $=$ mean humidity at $1400 \mathrm{~h}$.

b All coefficients were nonsignificant except where denoted by ${ }^{*}=$ significant at 0.05 and ${ }^{* *}=$ significant at 0.01 .

${ }^{\mathrm{c}}$ Since all accessions in Jordan were susceptible to stem rust, no correlation was necessary. 
ity. These significant correlations are not surprising given that Puccinia hordei requires moist conditions for infection and that high rainfall and humidity will increase disease pressure. However, $S$. passerinii, Pyrenophora teres f. teres, $C$. sativus, and Puccinia graminis f. sp. tritici also require moisture for infection, but no significant correlations were found to environmental parameters for these pathogens. There are several possible reasons for this result. For $S$. passerinii and Puccinia graminis f. sp. tritici, the lack of correlation between resistance and environmental parameters is likely related to the low degree of diversity found in the accessions for reaction to the two pathogens. Another possibility is that the general environmental data taken from weather stations near the collection sites may not be indicative of the micro-scale environment where $H$. spontaneum is subject to pathogen infection (e.g., in valleys or other water courses where moisture accumulates). It is also likely that the critical periods for pathogen infection and spread occur within short and specific time intervals, and that the environmental conditions that favor infection during these periods are not reflected in the values (e.g., average annual rainfall or humidity at 1400 hours, January temperature) that were used in this analysis. Environmental parameters such as leaf wetness duration, relative humidity, and temperature measured at the time of year that corresponds to critical plant growth stages (tillering to heading) may give a better indication of environmental influence on disease pressure, which is more likely to correlate to evolution of disease resistance. Due to the overall lack of correlation between resistance and environmental parameters assessed in this study, it would be difficult to reliably identify specific sites where possible sources of resistance to specific diseases might be found. However, this may be possible at a regional level. The frequency of resistance in $H$. spontaneum was generally higher in areas of greater precipitation. Thus, one may be more likely to find resistant $H$. spontaneum accessions in environments that are mesic (e.g., along the Mediterranean coast and Jordan River) than in those that are xeric (e.g., Negev Desert).

The frequency (77 to 98\%) and level (most with IR of 0) of resistance to SSLB in $H$. spontaneum was the highest among all diseases examined in this study. This was especially true for accessions from Israel. Resistance to SSLB appears to be very common in $H$. spontaneum, as many resistant accessions have been reported from other countries such as Iraq, Lebanon, Syria, and Turkey $(22,28,45) . \quad S$. passerinii has been reported on cultivated barley in Israel (19), but its role in the coevolution of $H$. spontaneum resistance is not known. It is possible that a higher level of variation for reaction to SSLB exists in
$H$. spontaneum but was simply not detected by the isolate of $S$. passerinii used in this study. Additional studies using isolates of $S$. passerinii from the Middle East and other regions should be conducted to obtain a more comprehensive assessment of the diversity in H. spontaneum to SSLB. It was interesting to note that accessions with SSLB resistance were found at several collection sites (e.g., Bor Mashash, Revivum, Yeroham, and Sede Boqer) in the Negev Desert, where precipitation is less than $200 \mathrm{~mm}$ per year. This result was unexpected given that $S$. passerinii is a splash-dispersed pathogen and requires moist weather conditions for infection and spread.

Several previous studies have found $H$. spontaneum to be a rich source of powdery mildew resistance $(7,15)$. Our data confirmed these findings, as 58 and $70 \%$ of accessions from Jordan and Israel, respectively, exhibited resistant infection responses. In this study, resistance was more common in mesic environments, although several highly resistant accessions were also identified in the xeric environments of Revivum and Machtesh-Gadol in the Negev Desert of Israel. This result is in contrast to previous research where powdery mildew resistance was absent at sites near the Negev Desert (31). The contrasting results found between this and the other two studies may be due to the different pathogen cultures used or perhaps a limited host sample size. Nevertheless, this indicates that sources of powdery mildew resistance can be found in areas where the environment might be considered unfavorable for the development of the disease. Fischbeck and Jahoor (11) studied the genetics of powdery mildew resistance in $H$. spontaneum accessions obtained from Israel and found that the resistance response was quantitative when challenged by local isolates of B. graminis f. sp. hordei, but qualitative (hypersensitive reactions) when challenged by European pathogen isolates. This finding has important implications regarding the choice of pathogen isolates used in disease screening tests. Future evaluations of this germ plasm should be made to local pathotypes isolated at or near sites where the $H$. spontaneum accessions were collected.

The percentage of $H$. spontaneum accessions exhibiting resistance to Puccinia hordei was high (70\%) and very high $(90 \%)$ in Jordan and Israel, respectively. The alternate host of Puccinia hordei is Ornithogalum (Star of Bethlehem lily), which is endemic to Israel and co-exists with $H$. spontaneum in many regions of the country (3). Strong co-evolutionary forces between the host and pathogen (as mediated by the alternate host) have been operating for several millennia and likely contributed to the great diversity found in $H$. spontaneum for leaf rust reaction. Leaf rust resistance was more common in accessions from collection sites with higher rainfall and humidity, as revealed in the correlation analysis. Anikster et al. (2) found that leaf rust resistance was common in $H$. spontaneum accessions from central and northern Israel, but not in the arid regions of the south. Similarly, Moseman et al. (30) found leaf rust resistance common in accessions from the Coastal Plains of Israel, where the environmental conditions are more conducive for disease development (i.e., higher rainfall and humidity) and Ornithogalum is present. Manisterski et al. (27) speculated that $H$. spontaneum originating from dry regions in Israel would likely be universally susceptible to leaf rust. However, in this study, we identified highly resistant accessions (IT of 0;) from the arid sites of Revivum and Sede Boqer in the northern Negev Desert. This finding highlights the great diversity for disease resistance in $H$. spontaneum across the center of origin (51). Our study also confirms and extends previous studies $(17,27,30)$ documenting the large reservoir of unexploited leaf rust resistance in this wild progenitor species. Indeed, four of the seven most recently described leaf rust resistance genes in Hordeum (Rph10, Rph11, Rph15, and Rph16) were derived from $H$. spontaneum (13). Future leaf rust research on $H$. spontaneum should include evaluations to more widely virulent cultures of Puccinia hordei and genetic tests to determine the allelic relationships of resistance genes to those already described.

About two-thirds of the $H$. spontaneum accessions evaluated in this study exhibited intermediate to high levels of resistance to Pyrenophora teres f. teres. Similar results were found for accessions from the Middle East by several other research groups $(14,22,28,38)$. Kenneth et al. (20) reported a high degree of diversity for net blotch reaction in $H$. spontaneum and for virulence in Pyrenophora teres f. teres in Israel. It is therefore likely that Israel is an important center of diversity for the pathogen and that co-evolutionary forces are operating in this pathosystem. In this study, accessions with high levels of net blotch resistance were found in the higher moisture areas of the Mediterranean coast to the arid region of the Negev Desert. It was interesting to note that the number of accessions that were susceptible to net blotch was high in the Golan Heights - a region where leaf rust and powdery mildew resistance was common.

In contrast to the diseases described above, the frequency of resistance to spot blotch in $H$. spontaneum was lower (46 to $53 \%$ ). Other investigators have reported a low frequency of spot blotch resistance in this species. This result is in agreement with previous investigations. Jana and Bailey (14) evaluated $291 \mathrm{H}$. spontaneum accessions to $C$. sativus and found only 13 with resistance ( $4.5 \%$ of total). Metcalfe et al. (28) and Legge et al. (22) evaluated a 
collection of 43 accessions from Turkey, Iraq, Lebanon, and Syria, but found no resistance. In this study, six accessions exhibited high levels of spot blotch resistance (IR $=2$ ), four from Israel (Gadot 635, Bet Shean 21-46, Mehola 22-42, and Avedat 33-28) and two from Jordan (Shūna N. 1-43 and Irbid 2-14). All but one (Avedat 33-28) of these accessions was from northern Israel and Jordan.

Resistance to stem rust was extremely rare in $H$. spontaneum accessions from Israel and Jordan. This result is in agreement with those of Metcalfe et al. (28) and Legge et al. (22), who found no promising levels of stem rust resistance in $H$. spontaneum from the Middle East. The alternate hosts of Puccinia graminis f. sp. tritici (i.e., Berberis and Mahonia spp.) originated in Asia $(3,23)$; thus, strong coevolutionary forces were apparently not operating between the pathogen and $H$. spontaneum in this region of the Fertile Crescent. Accessions from Damon, Gadot, and Shechem in Israel did exhibit high levels of resistance (ITs $=0 ; 1$ to 120 ;) to pathotypes MCCF and QCCJ, but only at the low-temperature environment. These accessions should be evaluated in the field to determine whether they carry broadbased adult plant resistance against Puccinia graminis f. sp. tritici pathotypes commonly found in North America.

One of the primary objectives of this research was to identify accessions of $H$. spontaneum that possessed high levels of resistance to all six pathogens of importance in the Upper Midwest region of the United States and central provinces of Canada. Such accessions could then be crossed with adapted breeding lines to simultaneously transfer genes for multiple disease resistance, possibly by markerassisted selection. Two of the 116 accessions (Shechem 12-32 and Damon 11-11) evaluated were resistant to all of the pathogens tested. These accessions were collected from fairly divergent sites in northeastern and north-central Israel. Only a small fraction $(\sim 3 \%)$ of the available accessions from the Institute of Evolution was evaluated in this study; thus, it is likely that additional sources of multiple disease resistance will be identified. Crosses have been made between the two $H$. spontaneum resistance sources and advanced barley breeding lines to elucidate the number, effect, and chromosomal position of the resistance genes by molecular mapping techniques. Identification of molecular markers closely linked to the resistance genes will facilitate their introgression in breeding lines by marker-assisted selection. Six-rowed malting barley cultivars in the Upper Midwest region of the United States have adequate levels of spot blotch resistance and stem rust resistance (except to pathotype QCCJ). The incorporation of SSLB, powdery mildew, leaf rust, and net blotch resistance will allow pro- ducers to attain more stable yields in their barley cultivars without the threat of disease losses.

\section{ACKNOWLEDGMENTS}

The authors gratefully acknowledge the assistance of Hala Toubia-Rahme for evaluating $H$. spontaneum accessions for resistance to Septoria speckled leaf blotch. We also thank Yongliang Sun, Renee Senn, Firas Abu-El Samen, and Faisal Nimer for their technical assistance in this study. A portion of this research was conducted at North Dakota State University by the first two authors. This research was funded in part by the American Malting Barley Association, the LiebermanOkinow Endowment at the University of Minnesota, and Agriculture and Agri-Food Canada.

\section{LITERATURE CITED}

1. Abbott, D. C., Brown, A. H. D., and Burdon, J. J. 1992. Genes for scald resistance from wild barley (Hordeum vulgare ssp. spontaneum) and their linkage to isozyme markers. Euphytica 61:225-231.

2. Anikster, Y., Moseman, J. G., and Wahl, I. 1976. Parasite specialization of Puccinia hordei Otth and sources of resistance in Hordeum spontaneum C. Koch. Pages 468-469 in: Proc. Int. Barley Genet. Sympos., 3rd. H. Gaul, ed. Garching, Germany.

3. Anikster, Y., and Wahl, I. 1979. Coevolution of the rust fungi on Gramineae and Liliaceae and their hosts. Annu. Rev. Phytopathol. 17:367-403.

4. Badr, A., Müller, K., Schäfer-Pregl, R., El Rabey, H., Effgen, S., Ibrahim, H. H., Pozzi, C., Rohde, W., and Salamini, F. 2000. On the origin and domestication history of barley (Hordeum vulgare). Mol. Biol. Evol. 17:499510.

5. Baek, H. J., Beharav, A., and Nevo, E. 2003. Ecological-genomic diversity of microsatellites in wild barley (Hordeum spontaneum) populations in Jordan. Theor. Appl. Genet. 106:397-410

6. Baum, B. R., Nevo, E., Johnson, D. A., and Beiles, A. 1997. Genetic diversity in wild barley (Hordeum spontaneum C. Koch) in the Near East: A molecular analysis using Random Amplified Polymorphic DNA (RAPD) markers. Gen. Res. Crop Evol. 44:147-157.

7. Dreiseitl, A., and Bockleman, H. E. 2000. Investigation of the wild barley germplasm collection for powdery mildew resistance. Pages 72-74 in: Proc. Int. Barley Genetics Sympos., 8th. Susan Logue, ed. Adelaide, Australia.

8. Fetch, T. G., Jr., and Steffenson, B. J. 1994. Identification of Cochliobolus sativus isolates expressing differential virulence on two-row barley genotypes from North Dakota. Can. J. Plant Pathol. 16:202-206.

9. Fetch, T. G., Jr., and Steffenson, B. J. 1999. Rating scales for assessing infection responses of barley infected with Cochliobolus sativus. Plant Dis. 83:213-217.

10. Fetch, T. G., Jr., Steffenson, B., and Nevo, E. 2000. Multiple disease resistance in Hordeum spontaneum accessions from Israel and Jordan. Pages 104-106 in: Proc. Int. Barley Genet. Sympos., 8th. Susan Logue, ed. Adelaide, Australia.

11. Fischbeck, G., and Jahoor, A. 1991. The transfer of genes for mildew resistance from Hordeum spontaneum. Pages 247-255 in: Integrated control of cereal mildews: Virulence patterns and their change. J. H. Jørgensen, ed. Ris $\varnothing$ Natl. Laboratory, Roskilde, Denmark.

12. Harlan, J. R. 1992. Crops \& Man. 2nd ed. American Society of Agronomy, Madison WI.

13. Ivandic, V., Walther, U., and Graner, A. 1998. Molecular mapping of a new gene in wild barley conferring complete resistance to leaf rust
(Puccinia hordei Otth). Theor. Appl. Genet. 97:1235-1239.

14. Jana, S., and Bailey, K. L. 1995. Responses of wild and cultivated barley from West Asia to net blotch and spot blotch. Crop Sci. 35:242246.

15. Jana, S., and Nevo, E. 1991. Variation in response to infection with Erysiphe graminis hordei and Puccinia hordei in some wild barley populations in a centre of diversity. Euphytica 57:133-140.

16. Jana, S., and Pietrzak, L. N. 1988. Comparative assessment of genetic diversity in wild and primitive cultivated barley in a center of diversity. Genetics 119:981-990.

17. Jin, Y., Steffenson, B. J., and Bockelman, H. E. 1995. Evaluation of cultivated and wild barley for resistance to pathotypes of Puccinia hordei with wide virulence. Gen. Res. Crop Evol. 42:1-6.

18. Jin, Y., Steffenson, B. J., and Miller, J. D. 1994. Inheritance of resistance to pathotypes QCC and MCC of Puccinia graminis f. sp. tritici in barley line Q21861 and temperature effects on the expression of resistance. Phytopathology 84:452-455.

19. Kenneth, R. 1960. Aspects of the taxonomy, biology, and epidemiology of Pyrenophor teres Drechsl. (Drechslera teres (Sacc.) Shoemaker), the causal agent of net blotch disease of barley. Ph.D. diss. The Hebrew University, Rehovot.

20. Kenneth, R., Atzmon, Y., Khair, J., Koltin, Y., and Wahl, I. 1967. Problems in breeding barley resistant to net blotch disease in Israel. Pages 46-47 in: Proc. Israel Congr. Plant Pathol., 1st. I. Wahl, ed. Rehovot, Israel.

21. Kølster, P., Munk, L., Stølen, O., and Løhde, J. 1986. Near-isogenic barley lines with genes for resistance to powdery mildew. Crop Sci. 26:903-907.

22. Legge, W. G., Metcalfe, D. R., Chiko, A. W., Martens, J. W., and Tekauz, A. 1996. Reaction of Turkish barley accessions to Canadian barley pathogens. Can J. Plant Sci. 76:927-931.

23. Leppik, E. E. 1970. Gene centers of plants as sources of disease resistance. Annu. Rev. Phytopathol. 8:323-344.

24. Levine, M. N., and Cherewick, W. J. 1952. Studies on dwarf leaf rust of barley. U.S. Dep. Agric. Tech. Bull. No. 1057.

25. Limpert, E., and Müller, K. 1994. Designations of pathotypes of plant pathogens. J. Phytopathol. 140:346-358.

26. Long, D. L., Steffenson, B. J., Leonard, K. J., Jin, Y., Hughes, M. E., and Casper, D. H. 1995. Barley rusts in the United States in 1995. Barley Newsl. Vol. 39. U.S. Dep. Agric. Published online.

27. Manisterski, J., Treeful, L., Tomerlin, J. R., Anikster, Y., Moseman, J. G., Wahl, I., and Wilcoxson, R. D. 1986. Resistance of wild barley accessions from Israel to leaf rust collected in the USA and Israel. Crop Sci. 26:727-730.

28. Metcalfe, D. R., Chiko, A. W., Martens, J. W. and Tekauz, A. 1977. Reaction of barleys from the Middle East to Canadian pathogens. Can. J. Plant Sci. 57:995-999.

29. Miller, J. D., and Lambert, J. W. 1955. Variability and inheritance of reaction of barley to race 15B of stem rust. Agron. J. 47:373-377.

30. Moseman, J. G., Nevo, E., and El-Morshidy, M. A. 1990. Reactions of Hordeum spontaneum to infection with two cultures of Puccinia hordei from Israel and United States. Euphytica 49:169-175.

31. Moseman, J. G., Nevo, E., and Zohary, D. 1983. Resistance of Hordeum spontaneum collected in Israel to infection with Erysiphe graminis hordei. Crop Sci. 23:1115-1119.

32. Nevo, E. 1992. Origin, evolution, population genetics and resources for breeding of wild 
barley, Hordeum spontaneum, in the Fertile Crescent. Pages 19-43 in: Barley: Genetics, Biochemistry, Molecular Biology and Biotechnology. Peter Shewry, ed. CAB International Press, London.

33. Nevo, E. 1998. Genetic diversity in wild cereals: Regional and local studies and their bearing on conservation ex situ and in situ. Gen. Res. Crop Evol. 45:355-370.

34. Nevo, E., Beiles, A., Gutterman, Y., Storch, N., and Kaplan, D. 1984. Genetic resources of wild cereals in Israel and vicinity. II. Phenotypic variation within and between populations of wild barley, Hordeum spontaneum. Euphytica 33:737-756.

35. Nevo, E., Moseman, J. G., Beiles, A., and Zohary, D. 1984. Correlation of ecological factors and allozymic variations with resistance to Erysiphe graminis hordei in Hordeum spontaneum in Israel: Patterns and application. Plant Syst. Evol. 145:79-96.

36. Nevo, E., Zohary, D., Brown, A. H. D., and Haber, M. 1979. Genetic diversity and environmental associations of wild barley, Hordeum spontaneum, in Israel. Evolution 33:815-833.

37. Roelfs, A. P., and Martens, J. W. 1988. An international system of nomenclature for $P u c$ cinia graminis f. sp. tritici. Phytopathology 78:526-533.

38. Sato, K., and Takeda, K. 1997. Net blotch resistance in wild species of Hordeum. Euphytica 95:179-185.

39. Steffenson, B. J., Hayes, P. M., and Kleinhofs, A. 1996. Genetics of seedling and adult plant resistance to net blotch (Pyrenophora teres $\mathrm{f}$. teres) and spot blotch (Cochliobolus sativus) in barley. Theor. Appl. Genet. 92:552-558.

40. Steffenson, B. J., Jin, Y., and Griffey, C. A. 1993. Pathotypes of Puccinia hordei with virulence for the barley leaf rust resistance gene Rph7 in the United States. Plant Dis. 77:867-869.

41. Steffenson, B. J., Jin, Y., Rossnagel, B. G., Rasmussen, J. B., and Kao, K. 1995. Genetics of multiple disease resistance in a doubledhaploid population of barley. Plant Breed. 114:50-54.

42. Steffenson, B. J., and Webster, R. K. 1992. Pathotype diversity of Pyrenophora teres $\mathrm{f}$. teres on barley. Phytopathology 82:170-177.

43. Tekauz, A. 1985. A numerical scale to classify reactions of barley to Pyrenophora teres. Can. J. Plant Pathol. 7:181-183.

44. Torp, J., Jensen, H. P., and Jørgensen, J. 1978. Powdery mildew resistance genes in 106 northwest European spring barley varieties. Pages 75-102 in: Yearbook Royal Vet. Agric. Univ. A. Klougart, ed. Copenhagen, Denmark.

45. Toubia-Rahme, H., and Steffenson, B. J. 1999. Sources of resistance to Septoria passerinii in Hordeum vulgare and $H$. vulgare subsp. spontaneum. Pages 156-158 in: Septoria and Stagonospora Diseases of Cereals: A Compilation of Global Research. M. van Ginkel, A. McNab, and J. Krupinsky, eds. CIMMYT, El-Batan, Mexico.

46. Turpeinen, T., Tenhola, T., Manninen, O., Nevo, E., and Nissila, E. 2001. Microsatellite diversity associated with ecological factors in
Hordeum spontaneum populations in Israel. Mol. Ecol. 10:1577-1591.

47. Valjavec-Gratian, M., and Steffenson, B. J. 1997. Pathotypes of Cochliobolus sativus on barley in North Dakota. Plant Dis. 81:12751278 .

48. Van Rijn, C. P. E., Heersche, I., Van Berkel, Y. E. M., Nevo, E., Lambers, H., and Poorter, H 2000. Growth characteristics in Hordeum spontaneum populations from different habitats. New Phytol. 146:471-481.

49. Van Valen, L. 1965. Morphological variation and width of ecological niche. Am. Naturalist 99:377-390.

50. Von Bothmer, R., Jacobsen, N., Baden, C., Jørgensen, R. B., and Linde-Laursen, I 1995. An Ecogeographical Study of the Genus Hordeum. 2nd ed. Systematic and Ecogeographic Studies on Crop Genepools 7. International Plant Genetic Resources Institute, Rome.

51. Wahl, I., Anikster, Y., Manisterski, J., and Segal, A. 1984. Evolution at the center of origin. Pages 39-77 in: The Cereal Rusts. Vol. I Origins, Specificity, Structure, and Physiology. W. R. Bushnell and A. P. Roelfs, eds. Academic Press, Orlando, FL.

52. Wu, H.-L., Steffenson, B. J., Li, Y., Oleson, A. E., and Zhong, S. 2003. Genetic variation for virulence and RFLP markers in Pyrenophora teres. Can. J. Plant Pathol. 25:82-90.

53. Zhukovsky, P. M. 1965. Main gene centers of cultivated plants and their wild relatives within the territory of the U.S.S.R. Euphytica 14:177-188. 